\title{
Cooperative Medium Access Control Based on Spectrum Leasing
}

\author{
3 Jiao Feng, Rong Zhang, Member, IEEE, Lajos Hanzo, Fellow, IEEE, and Soon Xin Ng, Senior Member, IEEE
}

\begin{abstract}
4 Abstract-Based on cooperative spectrum leasing, a distributed 5 "win-win" (WW) cooperative framework is designed to encourage 6 the licensed source node (SN) to lease some part of its spectral 7 resources to the unlicensed relay node $(\mathrm{RN})$ for the sake of si8 multaneously improving the SN's achievable rate and for reducing 9 the energy consumption (EC). The potential candidate RNs carry 10 out autonomous decisions concerning whether to contend for a 11 cooperative transmission opportunity, which could dissipate some 12 of their battery power, while conveying their traffic in light of their 13 individual service requirements. Furthermore, a WW cooperative 14 medium-access-control (MAC) protocol is designed to implement 15 the proposed distributed WW cooperative framework. Simulation 16 results demonstrate that our WW cooperative MAC protocol is 17 capable of providing both substantial rate improvements and 18 considerable energy savings for the cooperative spectrum leasing 19 system.
\end{abstract}

20 Index Terms-Author, please supply index terms/keywords 21 for your paper. To download the IEEE Taxonomy go to 22 http://www.ieee.org/documents/taxonomy_v101.pdf.

\section{INTRODUCTION}

24 OOPERATIVE communications techniques have recently 25 attracted substantial research attention [1] as a benefit 26 of their significant throughput improvements, energy savings, 27 and coverage enhancements. However, these benefits may be 28 eroded by the conventional higher layer protocols, which were 29 designed for classic noncooperative systems. Hence, it is im30 portant to design appropriate medium-access-control (MAC) 31 protocols to support cooperative physical layer techniques.

32 In contrast with the legacy wireless MAC protocols, cooper33 ative MAC protocols aim to cooperatively schedule the medium 34 access of all nodes while allowing the relay nodes (RNs) to 35 buffer and forward the others' data frames using the broadcast 36 nature of the wireless network, instead of ignoring these data 37 frames. There are numerous contributions in the literature on 38 designing cooperative MAC protocols, most of which aim to

Manuscript received December 11, 2012; revised April 28, 2013; accepted July 4, 2013. This work was supported in part by the Research Councils UK under the auspices of the U.K.-India Advanced Technology Center in Wireless Communications, the European Union CONCERTO Project, and the European Research Council's Advanced Fellow Grant and in part by the Royal Society's Wolfson Research Merit Award. The review of this paper was coordinated by Prof. J. Tang.

The authors are with the School of Electronics and Computer Science, University of Southampton, Southampton SO17 1BJ, U.K. (e-mail: 1h@ecs. soton.ac.uk).

Color versions of one or more of the figures in this paper are available online at http://ieeexplore.ieee.org.

Digital Object Identifier 10.1109/TVT.2013.2272895 maximize the throughput [2]-[6], including the widely recog- 39 nized CoopMAC of [7]. However, a potential impediment of the 40 CoopMAC is that its energy efficiency was traded off against 41 the throughput benefits claimed. Therefore, [8]-[12] aimed to 42 minimize the energy consumption (EC) by developing energy- 43 efficient cooperative MAC protocols. To jointly consider these 44 conflicting design objectives, Luo et al. [13] and Zhou et al. 45 [14] designed meritorious algorithms to improve the achievable 46 throughput and to simultaneously enhance the energy efficiency 47 achieved.

However, the aforementioned cooperative MAC protocols, 49 such as CoopMAC, were developed based on the common 50 assumption that the relays agree to altruistically forward the 51 data of the source node (SN). This unconditional altruistic 52 behavior is unrealistic to expect from mobile stations. In fact, 53 a greedy $\mathrm{RN}$ behavior is likely to be the norm in spectrum 54 leasing [15], where the licensed SN intends to lease some part 55 of its spectral resources to the unlicensed $\mathrm{RN}$ in exchange for 56 appropriate "remuneration." In this spectrum leasing system, 57 the unlicensed RNs also have an incentive to support the SN 58 to achieve its quality-of-service (QoS) target in exchange for a 59 transmission opportunity. This cooperation allows both the SN 60 and the RN to satisfy its individual requirement. Based on this 61 cooperative spectrum leasing system, some early theoretical 62 studies have been conducted in [16]-[21]. Bearing in mind the 63 greedy behavior of the mobile RNs, meritorious game-theoretic 64 frameworks were proposed in [16]-[19] to maximize the SN's 65 transmit rate while simultaneously satisfying the requirements 66 of the RNs. Based on game theory, Hafeez and Elmirghani 67 [20] and Jayaweera et al. [21] aimed to minimize the $E C$ of 68 cooperative spectrum leasing systems by designing beneficial 69 game-aided strategies. However, the joint optimization of the 70 transmit rate and of the $\mathrm{EC}$ has not been considered in these 71 existing works. Furthermore, the design of an appropriate 72 cooperative MAC protocol for practically implementing the 73 theoretical framework was not discussed in [16]-[21]. 74

Against this backdrop, the contributions of this paper are as 75 follows.

1) We first formulate a distributed "win-win" (WW) coop- 77 erative framework (DWWCF) to encourage the SN to 78 lease part of its spectral resources to the unlicensed RN 79 for the sake of improving the SN's transmit rate and for 80 simultaneously reducing the SN's $E C$ while ensuring that 81 the unlicensed RNs are capable of securing a transmission 82 opportunity for their own traffic and for satisfying their 83 QoS. Furthermore, the proposed DWWCF selects the 84 

121 network's architecture and our DWWCF are introduced in 122 Section II. Section III describes the proposed WW cooperative 123 MAC protocol, whereas in Section IV, the attainable perfor124 mance of our scheme is quantified. Finally, we conclude in 125 Section V.

\section{System Model ANd Distributed Win-Win COOPERATIVE FRAMEWORK}

\section{A. System Model}

129 Before embarking on outlining our DWWCF, we introduce 130 our network topology and outline our assumptions.

131 As shown in Fig. 1, we consider a cooperative network 132 having a single $\mathrm{SN} \mathcal{S}$ and a total of $N$ RNs in the set $\mathcal{R}=$ $133\left\{\mathcal{R}_{1}, \ldots, \mathcal{R}_{N}\right\}$, as well as a common DN $\mathcal{D}$, where $\mathcal{D}$ may be 134 a base station (BS) or an ad hoc cluster head. Both $\mathcal{S}$ and $\mathcal{D}$ are 135 granted access to the licensed spectrum, whereas the $N$ RNs 136 are not licensees. To simplify our investigations, we made the 137 following assumptions. All the channels involved are assumed 138 to undergo quasi-static Rayleigh fading; hence, the complex139 valued fading envelope remains constant during a transmission

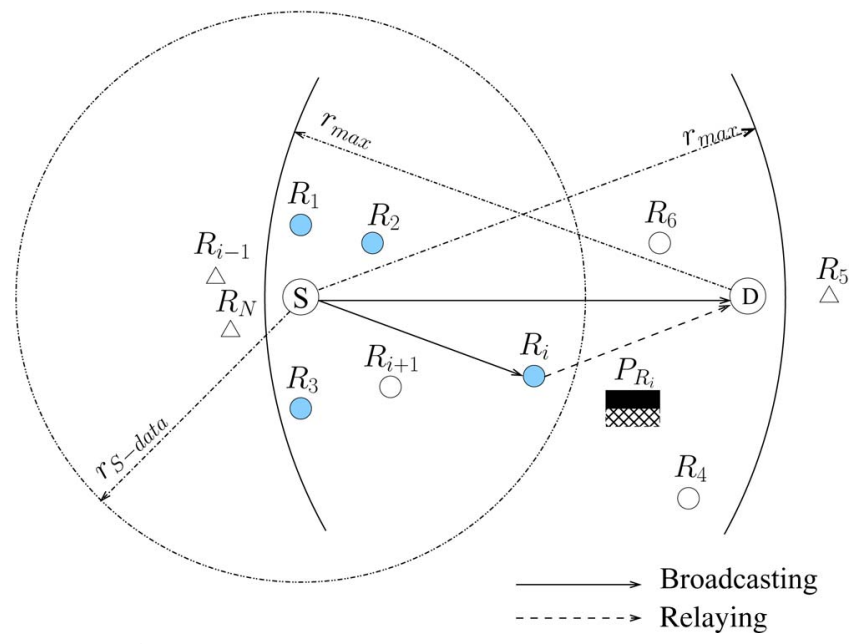

Fig. 1. Cooperative topology consists of one $\mathrm{SN} \mathcal{S}$, one $\mathrm{DN} \mathcal{D}$, and a total of $N$ RNs $\mathcal{R}=\left\{\mathcal{R}_{1}, \ldots, \mathcal{R}_{N}\right\}$.

burst, ${ }^{1}$ whereas it is faded independently between the consec- 140 utive transmission bursts. Within a given transmission burst, 141 the duplex bidirectional channels between a pair of actively 142 communicating nodes are assumed to be identical, whereas 143 the channels of any of the remaining links are independent. 144 We assume perfect channel estimation for all nodes concerning 145 their own channels, ${ }^{2}$ but no knowledge of the remaining links 146 is assumed. Additionally, the nodes' own position information 147 is perfectly known at each node. We consider the effects of 148 free-space path loss that is modeled by $\rho=\lambda^{2} / 16 \pi^{2} d^{\eta}$, where 149 $\lambda$ represents the wavelength, $d$ is the transmitter-to-receiver 150 distance and $\eta$ denotes the path-loss exponent, which is 2 . All 151 nodes are assumed to be limited by the same maximum transmit 152 power $P_{\max }$.

\section{B. Distributed WW Cooperative Framework}

1) SN's Behavior: Rather than relying on monetary remu- 155 neration, $\mathcal{S}$ in our DWWCF intends to lease part of its spec- 156 trum to the RNs in exchange for cooperatively supporting 157 the source's transmission. Based on the RN's assistance, $\mathcal{S} 158$ is capable of successfully conveying its data at a reduced 159 transmit power of $P_{\mathcal{S} \text {-data }}$ and an increased transmit rate 160 of $\alpha C_{\mathcal{S}, \mathcal{D}}^{\max }(\alpha \geq 1)$, which is the SN's target transmit rate. 161 In greater detail, $\alpha$ is the ratio of the desired and afford- 162 able throughput termed as the SN's "factor of greediness," 163 whereas $C_{\mathcal{S}, \mathcal{D}}^{\max }$ is the maximum achievable rate of the source- 164 to-destination (SD) link, which can be formulated as $C_{\mathcal{S}, \mathcal{D}}^{\max }=165$ $\log _{2}\left(1+\left(\rho_{\mathcal{S}, \mathcal{D}}\left|h_{\mathcal{S}, \mathcal{D}}\right|^{2} P_{\max } / P_{N}\right)\right)$, where $P_{N}$ is the power of 166 the additive white Gaussian noise, whereas $\left|h_{\mathcal{S}, \mathcal{D}}\right|$ denotes the 167 magnitude of the flat Rayleigh channel between $\mathcal{S}$ and $\mathcal{D}$. Fur- 168 thermore, $\rho_{\mathcal{S}, \mathcal{D}}$ is the free-space path-loss gain between $\mathcal{S}$ and 169 $\mathcal{D}$. If $\mathcal{S}$ cannot acquire any cooperative transmission assistance, 170 it directly transmits its data to $\mathcal{D}$ at a higher transmit power 171

\footnotetext{
${ }^{1}$ We define a transmission burst as a single transmission attempt, excluding any subsequent retransmission attempts.

${ }^{2}$ The effect of realistic imperfect channel estimation is evaluated in Section IV-F
} 
$172 P_{\mathcal{S}}^{n c}$ and lower transmit rate $R_{\mathcal{S}}^{n c}$. Hence, $\mathcal{S}$ has two Objective 173 Functions (OF) in our DWWCF, which may be formulated as

$$
\begin{aligned}
& \mathrm{OF}_{\mathcal{S} 1}=\max \left\{\xi_{\mathcal{S}} \cdot R_{\mathcal{S}}^{r e q}+\left(1-\xi_{\mathcal{S}}\right) \cdot R_{\mathcal{S}}^{n c}\right\} \\
& \mathrm{OF}_{\mathcal{S} 2}=\min \left\{\xi_{\mathcal{S}} \cdot P_{\mathcal{S}-\text { data }}+\left(1-\xi_{\mathcal{S}}\right) \cdot P_{\mathcal{S}}^{n c}\right\}
\end{aligned}
$$

174 subject to $R_{\mathcal{S}}^{r e q}=\alpha C_{\mathcal{S}, \mathcal{D}}^{\max }>R_{\mathcal{S}}^{n c}$ and $\alpha \geq 1$, as well as $175 P_{\mathcal{S} \text {-data }}<P_{\mathcal{S}}^{n c}$, where $\xi_{\mathcal{S}}$ denotes the cooperative probability 176 of SN.

177 2) RN's Behavior: According to our DWWCF, the RN has 178 an incentive to forward data for $\mathcal{S}$ for the sake of accessing 179 the SN's spectrum to convey its own traffic. The selfish RN $\mathcal{R}_{i}$ 180 reserves a certain fraction of $\beta C_{\mathcal{R}_{i}, \mathcal{D}}^{\max }(0<\beta<1)$ of the Relay181 to-Destination (RD) channel's capacity for conveying its own 182 traffic, where $\beta$ is the RN's "factor of greediness" and $C_{\mathcal{R}_{i}, \mathcal{D}}^{\max }$ is 183 given by: $C_{\mathcal{R}_{i}, \mathcal{D}}^{\max }=\log _{2}\left(1+\left(\rho_{\mathcal{R}_{i}, \mathcal{D}}\left|h_{\mathcal{R}_{i}, \mathcal{D}}\right|^{2} P_{\max } / P_{N}\right)\right)$, while $184\left|h_{\mathcal{R}_{i}, \mathcal{D}}\right|$ denotes the magnitude of the flat Rayleigh channel 185 between $\mathcal{R}_{i}$ as well as $\mathcal{D}$, and $\rho_{\mathcal{R}_{i}, \mathcal{D}}$ is the free-space path186 loss gain between $\mathcal{R}_{i}$ and $\mathcal{D}$. Based on our DWWCF, each $187 \mathrm{RN} \mathcal{R}_{i}$ carries out autonomous decisions concerning its own 188 cooperative strategy by optimizing its own OF, which may be 189 formulated as

$$
\mathrm{OF}_{\mathrm{RN} 1}=\max \left\{\xi_{\mathcal{R}_{i}} \cdot \beta C_{\mathcal{R}_{i}, \mathcal{D}}^{\max }\right\}
$$

190 subject to $0<\beta<1$, where $\xi_{\mathcal{R}_{i}}$ denotes the probability that $191 \mathrm{RN} \mathcal{R}_{i}$ is granted the transmission opportunity.

192 When the RNs provide cooperative transmission assis193 tance, extra energy is dissipated when relaying data for $\mathcal{S}$. 194 Hence, another OF is designed in our DWWCF to select the 195 best RN, which may be formulated as

$$
\mathrm{OF}_{\mathrm{RN} 2}=\min \sum_{i=1}^{N}\left\{\xi_{\mathcal{R}_{i}} \cdot P_{\mathcal{R}_{i}}\right\}
$$

196 subject to $\sum_{i=1}^{N} \xi_{\mathcal{R}_{i}} \leq 1$, and $P_{\mathcal{R}_{i}} \leq P_{\max }$, where $P_{\mathcal{R}_{i}}$ is the 197 RN's transmit power required for successfully forwarding the 198 SN's data and for simultaneously conveying its own data. Based 199 on the above OFs, it is quite a challenge to mathematically 200 solve these optimization problems in our DWWCF. Hence, we 201 designed a WW cooperative MAC protocol to implement our 202 DWWCF.

\section{III. Win-Win Cooperative Medium Access Control 204 PROTOCOL DESCRIPTION}

205 Based on the request-to-send/clear-to-send (RTS/CTS) sig206 naling of the legacy IEEE 802.11 protocol, a WW cooperative 207 MAC protocol is developed to implement our DWWCF, which 208 is formulated in Section II-B. The proposed signaling procedure 209 is detailed in Fig. 2, which includes three phases, as detailed in 210 the following.

\section{A. Phase I: Initialization}

212 Before $\mathcal{S}$ transmits any data frame, it issues an RTS message 213 to $\mathcal{D}$ at the maximum transmission power $P_{\max }$ to reserve the 214 shared channel, as shown in Fig. 2 . When $\mathcal{D}$ correctly receives 215 the RTS message, it replies with a CTS message, employing the

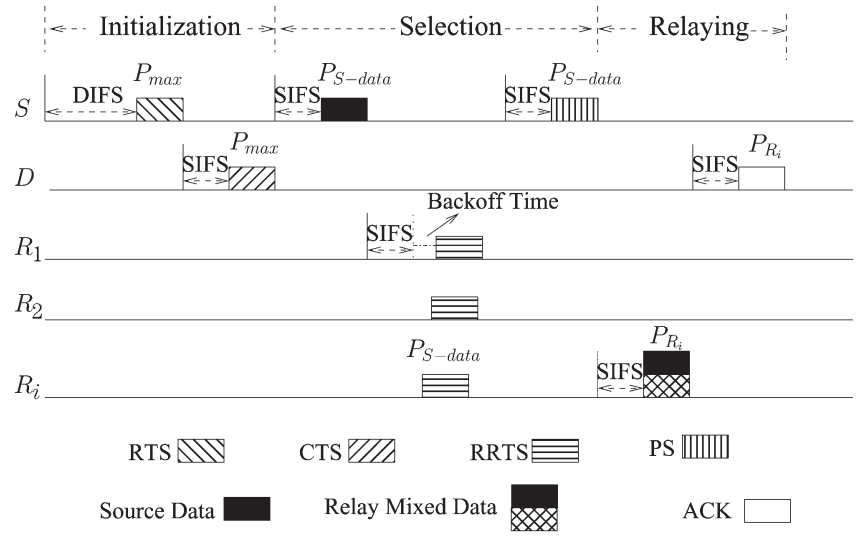

Fig. 2. Overall signaling procedure. RTS: Request-to-send. CTS: Clear-tosend. RRTS: Relay-request-to-send. PS: Please-send. ACK: Acknowledgement. DIFS: Distributed interframe space. SIFS: Short interframe space.

same transmission power $P_{\max }$. The instantaneous transmission 216 ranges of the sources are shown in Fig. 1. To elaborate a little 217 further, we include the transmitter's position information into 218 the RTS and CTS signaling frame; thus, any RNs in the set $\mathcal{R}, 219$ which can overhear both the RTS and CTS messages, will be 220 aware of the imminently forthcoming transmission and of the 221 position information on $\mathcal{S}$ and $\mathcal{D}$. Based on the knowledge of 222 their own position and on the position of the $\mathrm{SN}$ and the DN, 223 these RNs are capable of calculating the distances from both the 224 $\mathrm{SN}$ and the DN to themselves. These RNs, which are denoted 225 by filled or hollow circles in Fig. 1, form a potential cooperative 226 $\mathrm{RN}$ set $\mathcal{R}_{c} \subset \mathcal{R}$.

\section{B. Phase II: Relay Selection}

Following the initialization phase, the RN selection proce- 229 dure is constituted by a data transmission and two beacon 230 message exchanges, as detailed in the following.

231

1) Step I-Invitation for Cooperation: If $\mathcal{S}$ does not receive 232 a CTS message from $\mathcal{D}$, it would retransmit the RTS message as 233 specified in the legacy IEEE 802.11 protocol [22]. In contrast, if 234 $\mathcal{S}$ receives a CTS message from $\mathcal{D}$, it broadcasts its data frame 235 after a short interframe space (SIFS) interval at reduced power 236 of $P_{\mathcal{S} \text {-data }}$ and its target transmit rate of $\alpha C_{\mathcal{S}, \mathcal{D}}^{\max }(\alpha \geq 1)$, as 237 shown in Fig. 2. As a result, both $\mathcal{D}$ and the RNs in the set 238 $\mathcal{R}_{c}$ will hear this broadcast. When $\alpha$ is higher than unity, the 239 SN's data cannot be successfully transmitted to $\mathcal{D}$ in its entirety. 240 However, $\mathcal{D}$ will store this data frame and exploits the classic 241 Chase combining scheme [23] to combine it with the duplicated 242 data frame independently transmitted by the potential candidate 243 relays, for the sake of achieving rate improvements. Therefore, 244 the SN's aggregated rate achieved by using Chase combining 245 may be expressed as [24]

$$
\alpha C_{\mathcal{S}, \mathcal{D}}^{\max }=\log _{2}\left(1+\gamma_{\mathcal{S}, \mathcal{D}}^{(1)}+\gamma_{\mathcal{R}_{i}}^{\mathcal{S}}\right)
$$

subject to $\alpha \geq 1$, where $\gamma_{\mathcal{S}, \mathcal{D}}^{(1)}$ denotes the receiver's signal- 247 to-interference-plus-noise ratio (SINR) related to the direct 248 transmission during the broadcast phase. Furthermore, $\gamma_{\mathcal{R}_{i}}^{\mathcal{S}} 249$ represents the receive SINR of the SN's data frame, which is 250 transmitted during the relaying phase to be introduced. Based 251 


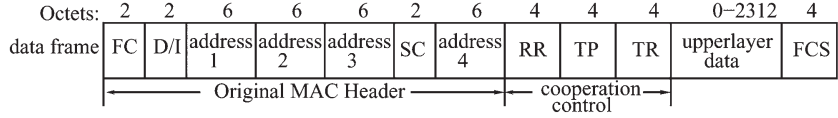

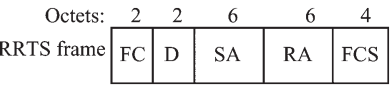

$$
\begin{aligned}
& \begin{array}{rl|l|l|l|}
\text { Octets: } & 2 & 2 & 6 & 4 \\
\text { PS frame } & \text { FC } & \text { D } & \text { BRA } & \text { FCS } \\
\hline
\end{array}
\end{aligned}
$$

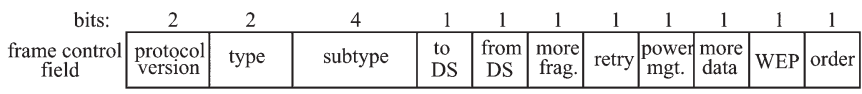

$$
\begin{aligned}
& \mathrm{FC}=\text { frame control } \quad \mathrm{D} / \mathrm{I}=\text { duration } / \mathrm{ID} \quad \mathrm{SC}=\text { sequence control } \quad \mathrm{RR}=\text { relay requirement } \\
& \mathrm{TP}=\text { transmit power } \quad \mathrm{TR}=\text { transmit rate } \mathrm{FCS}=\text { frame check sequence } \mathrm{D}=\text { duration } \\
& \mathrm{DS}=\text { distribution system } \mathrm{RA}=\text { relay address } \quad \mathrm{SA}=\text { source address } \quad \mathrm{BRA}=\text { best relay address }
\end{aligned}
$$

Fig. 3. Formats of the data frames, the RRTS message, and the PS message.

252 on the estimated channel state information (CSI) of the SD 253 link, $\mathcal{S}$ first calculates the receive SINR of $\gamma_{\mathcal{S}, \mathcal{D}}^{(1)}$ achieved by 254 the direct transmission during the broadcast phase. Then, based 255 on $\gamma_{\mathcal{S}, \mathcal{D}}^{(1)}$ and (5), $\mathcal{S}$ calculates the receive SINR of $\gamma_{\mathcal{R}}^{\mathcal{S}}$, which 256 must be guaranteed by the best $\mathrm{RN}$ and includes the value of $257 \gamma_{\mathcal{R}_{i}}^{\mathcal{S}}$ into the relay requirement (RR) field of its data frame for 258 implicitly informing the RNs of the SN's transmit requirement $259 \alpha C_{\mathcal{S}, \mathcal{D}}^{\max }$. The RNs in the vicinity, which correctly receive the 260 SN's data frame, are capable of inferring the value of $\gamma_{\mathcal{R}_{i}}^{\mathcal{S}}$ by 261 reading the RR field of the appropriately designed cooperative 262 MAC data frame, as shown in Fig. $3 .^{3}$

263 2) Step II-Contend for Cooperation: For clarity, we break 264 the discussion of this step into several subtopics, namely, the 265 cooperative decision, the backoff algorithm, and contention 266 message derivation.

267 Cooperation decision: If a particular $\mathrm{RN} \mathcal{R}_{i} \in \mathcal{R}_{c}$ erroneously 268 receives the data frame from $\mathcal{S}, \mathcal{R}_{i}$ would drop this data 269 frame and would keep on sensing the channel, as shown in Table I. On the other hand, if cooperative $\mathrm{RN} \mathcal{R}_{i} \in$ $\mathcal{R}_{c}$ correctly receives a data frame from $\mathcal{S}$, it calculates the transmit power $P_{\mathcal{R}_{i}}^{\mathcal{S}}$ necessitated to satisfy the $\mathrm{SN}$ rate requirement and the transmit power $P_{\mathcal{R}_{i}}^{\mathcal{R}}$ required to guarantee a throughput of $\beta C_{\mathcal{R}_{i}, \mathcal{D}}^{\max }$. If the sum of transmit power $P_{\mathcal{R}_{i}}=P_{\mathcal{R}_{i}}^{\mathcal{S}}+P_{\mathcal{R}_{i}}^{\mathcal{R}}$ is higher than $P_{\text {max }}, \mathcal{R}_{i}$ has to give up contending for the cooperative opportunity and drop this SN's data frame, as shown in Table I. On the other hand, if $P_{\mathcal{R}_{i}}$ does not exceed $P_{\max }, \mathcal{R}_{i}$ would send a relay-request-to-send (RRTS) message to $\mathcal{S}$ after waiting for a SIFS interval and its backoff time, which is calculated based on the proposed backoff algorithm for the sake of contending for a transmission opportunity, as shown in Table I. The RRTS message in Fig. 2 informs $\mathcal{S}$ about the RN's correct reception and its intention to cooperate. Hence, the specific RNs, which decide to contend for the transmission opportunity form a smaller contending set of $\mathcal{R}_{c c} \in \mathcal{R}_{c}$. These RNs are represented by the filled circles in Fig. 1. It is noted that the value of $P_{\mathcal{R}_{i}}$ is not included in the RRTS message in Fig. 3 since the proposed backoff

\footnotetext{
${ }^{3}$ Apart from the cooperative control fields of the data frame, as shown in Fig. 3, the remaining fields are the same as those of the data frame specified in the IEEE 802.11 standards [22].
}

TABLE I

Procedure of THE RN SUbMission CoOperative DeCISION

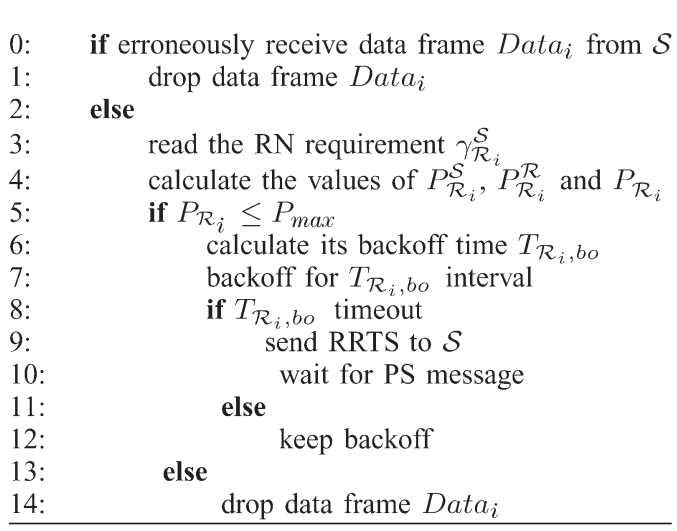

algorithm can identify the different values of $P_{\mathcal{R}_{i}}$ promised 290 by the contending RNs.

291

Backoff algorithm: To minimize the total transmit power of 292 the RNs, which is formulated by (4), we design a backoff 293 algorithm to select the best RN. As shown in Fig. 2, before 294 issuing the RRTS message, the RN $\mathcal{R}_{i} \in \mathcal{R}_{c c}$ has to wait 295 for a SIFS interval and for subsequent backoff duration 296 of $T_{\mathcal{R}_{i}}$,bo, which is defined as $T_{\mathcal{R}_{i}, \text { bo }}=\varphi_{\mathcal{R}_{i}} T_{w}$, where 297 $T_{w}=\mathrm{CWmin} \cdot$ SlotTime is the contention window $(\mathrm{CW}) 298$ length, ${ }^{4}$ with $\mathrm{CWmin}$ being the minimum $\mathrm{CW}$ duration 299 specified in the IEEE802.11 standards [22]. The coefficient 300 $\varphi_{\mathcal{R}_{i}}$ is defined as $\varphi_{\mathcal{R}_{i}}=P_{\mathcal{R}_{i}^{\min }} / P_{\max }$. Hence, the specific 301 candidate RN, which promises the lowest transmit power, 302 may first transmit its RRTS message as a benefit of its 303 shortest backoff time. In each RN selection phase, $\mathcal{S}$ has 304 to wait for a fixed period of ( $T_{w}+$ SlotTime $)$ to collect the 305 responses of the potential candidate RNs. If $\mathcal{S}$ correctly re- 306 ceives the RRTS message before its fixed waiting duration 307 times out, it selects the transmitter of that specific RRTS, 308 which was the first one to be correctly received as the 309 best RN, without considering the RRTS messages arriving 310 later and without comparing the specific transmit power 311 promised by the individual candidate RNs. Hence, the best 312 $\mathrm{RN}$ is selected in a distributed manner both without a cen- 313 tralized controller and without any information exchange 314 between the candidate RNs. Since the value of $P_{\mathcal{R}_{i}^{\min }} 315$ promised by the candidate $\mathrm{RN} \mathcal{R}_{i}$ is always lower than 316 $P_{\max }$, the backoff time allocated to $\mathcal{R}_{i}$ will not exceed the 317 SN's fixed waiting duration of ( $T_{w}+$ SlotTime $)$. Hence, all 318 the candidate RNs may issue their RRTS messages before 319 $\mathcal{S}$ stops waiting for the responses.

Contention message derivation: According to our backoff al- 321 gorithm, the specific RN promising the lowest power may 322 be granted the transmission opportunity to minimize the 323 total transmit power of RNs. Hence, the greedy RN has 324 to minimize its transmit power by only satisfying its rate 325 requirement of $\beta C_{\mathcal{R}_{i}, \mathcal{D}}^{\max }$ to wait for a shorter backoff time, 326

\footnotetext{
${ }^{4}$ In the IEEE 802.11 standard, a SlotTime consists of the time required to physically sense the medium and to declare the channel as "clear," as well as the MAC processing delay, the propagation delay, and the "receiver/transmitter turn-around time," which is the time required for the physical layer to change from receiving to transmitting at the start of the first bit [22].
} 
which is calculated based on the proposed backoff algorithm. Therefore, we have

$$
P_{\mathcal{R}_{i}^{\min }}\left(P_{\mathcal{R}_{i}}^{\mathcal{S}}, P_{\mathcal{R}_{i}^{\min }}^{\mathcal{R}} \mid \alpha, \beta\right)=P_{\mathcal{R}_{i}}^{\mathcal{S}}+P_{\mathcal{R}_{i}^{\min }}^{\mathcal{R}}
$$

subject to the condition of $C_{\mathcal{R}_{i}}^{\mathcal{R}}=\beta C_{\mathcal{R}_{i}, \mathcal{D}}^{\max }$ and $\alpha>1$, as well as $0<\beta<1$.

331 Let us now consider how to find $P_{\mathcal{R}_{i}}^{\mathcal{S}}$ and $P_{\mathcal{R}_{i}^{\min }}^{\mathcal{R}}$ of (6). In 332 our design, the RN employs SPC for jointly encoding both the 333 SN's and its own data. $\mathcal{D}$ then extracts the SN's data from 334 the relayed composite signal with the aid of SIC. Finally, the 335 extracted relayed component and the direct component are 336 combined. Assuming that $\mathcal{D}$ treats the RN's data frame as 337 interference, the receive SINR $\gamma_{\mathcal{R}_{i}}^{\mathcal{S}}$ of the SN's data frame re338 layed by the $\mathrm{RN}$ is given by $\gamma_{\mathcal{R}_{i}}^{\mathcal{S}}=\left(\rho_{\mathcal{R}_{i}, \mathcal{D}}\left|h_{\mathcal{R}_{i}, \mathcal{D}}\right|^{2} P_{\mathcal{R}_{i}}^{\mathcal{S}}\right) /\left(P_{N}+\right.$ $\left.339 \rho_{\mathcal{R}_{i}, \mathcal{D}}\left|h_{\mathcal{R}_{i}, \mathcal{D}}\right|^{2} P_{\mathcal{R}_{i}}^{\mathcal{R}}\right)$. After successfully retrieving the SN's data 340 frame, $\mathcal{D}$ becomes capable of decoding the RN's data frame by 341 removing the SN's interference with the aid of a SIC scheme 342 [25]. Hence, the achievable rate of the RN may be formulated as $343 C_{\mathcal{R}_{i}}^{\mathcal{R}}=\log _{2}\left(1+\left(\rho_{\mathcal{R}_{i}, \mathcal{D}}\left|h_{\mathcal{R}_{i}, \mathcal{D}}\right|^{2} P_{\mathcal{R}_{i}}^{\mathcal{R}} / P_{N}\right)\right)$. According to the 344 relaying strategy employed, the RN calculates the minimum 345 power required for the rate $C_{\mathcal{R}_{i}}^{\mathcal{R}}$ to reach $\beta C_{\mathcal{R}_{i}, \mathcal{D}}^{\max }$. Thus, the 346 value of $P_{\mathcal{R}_{i}^{\min }}^{\mathcal{R}}$ is explicitly given as $P_{\mathcal{R}_{i}^{\min }}^{\mathcal{R}}=\left(\left(2^{\beta C_{\mathcal{R}_{i}, \mathcal{D}}^{\max }}\right.\right.$ $\left.3471) P_{N}\right) /\left(\rho_{\mathcal{R}_{i}, \mathcal{D}}\left|h_{\mathcal{R}_{i}, \mathcal{D}}\right|^{2}\right)$, which is subjected to $0<\beta<1$. 348 Likewise, based on the metrics of $\gamma_{\mathcal{R}_{i}}^{\mathcal{S}}$ and $P_{\mathcal{R}_{i}^{\min }}^{\mathcal{R}}$, the RN 349 is capable of calculating the transmit power $P_{\mathcal{R}_{i}}{ }^{i}$ required for 350 successfully delivering the SN's data at a throughput of $\alpha C_{\mathcal{S}, \mathcal{D}}^{\max }$, 351 which is given by $P_{\mathcal{R}_{i}}^{\mathcal{S}}=\gamma_{\mathcal{R}_{i}}^{\mathcal{S}}\left(\left(P_{N} / \rho_{\mathcal{R}_{i}, \mathcal{D}}\left|h_{\mathcal{R}_{i}, \mathcal{D}}\right|^{2}\right)+P_{\mathcal{R}_{i}^{\min }}^{\mathcal{R}}\right)$, 352 where $\gamma_{\mathcal{R}_{i}}^{\mathcal{S}}$ has been given in Step I. Based on the given 353 derivation, $\mathcal{R}_{i}$ calculates the value of $P_{\mathcal{R}_{i}}^{\min }$ as the sum of $P_{\mathcal{R}_{i}}^{\mathcal{S}}$ 354 and $P_{\mathcal{R}_{i}^{\min }}^{\mathcal{R}}$.

355 3) Step III-Accept for Cooperation: After waiting for the 356 fixed duration of $\left(T_{w}+\right.$ SlotTime $)$ specified by the proposed 357 backoff algorithm and for a subsequent SIFS interval, $\mathcal{S}$ replies 358 to the best RN $\mathcal{R}_{\hat{i}}$ associated with the first RRTS message that 359 was correctly received by sending a please-send (PS) message if $360 \mathcal{S}$ correctly received the RRTS message during its fixed waiting 361 period of $\left(T_{w}+\right.$ SlotTime $)$, as shown in Fig. 2 and Table II. The 362 format of the PS frame is characterized in Fig. 3. Since the SN 363 sends its data frame and PS message at the same transmission 364 power of $P_{\mathcal{S} \text {-data }}$, all the RNs, which have correctly received 365 the data frame from the SN will overhear the PS message. This 366 guarantees that only the best $\mathrm{RN}$ forwards its data frame to $\mathcal{D}$ 367 during the data-forwarding phase.

\section{C. Phase III: Cooperative Transmission}

369 In this phase, the best RN $\mathcal{R}_{\hat{i}}$ forwards the superimposed SR 370 data to $\mathcal{D}$ if $\mathcal{S}$ successfully selects the best RN. Otherwise, $\mathcal{S}$ 371 retransmits its data frame to $\mathcal{D}$, as shown in Fig. 2 and Table II. 372 1) Data Forwarding and Relay Retransmission: If RN $\mathcal{R}_{i} \in$ $373 \mathcal{R}_{c c}$ finds that the receiver of the received PS message is not 374 itself, it would drop the SN's data and would keep on sensing 375 the medium. On the other hand, if the $\mathrm{RN} \mathcal{R}_{i} \in \mathcal{R}_{c c}$ received 376 a PS message that is destined for itself, it will encode both the 377 SN's and its data with the aid of SPC and will forward the super-
TABLE II

PROCEDURE OF SN

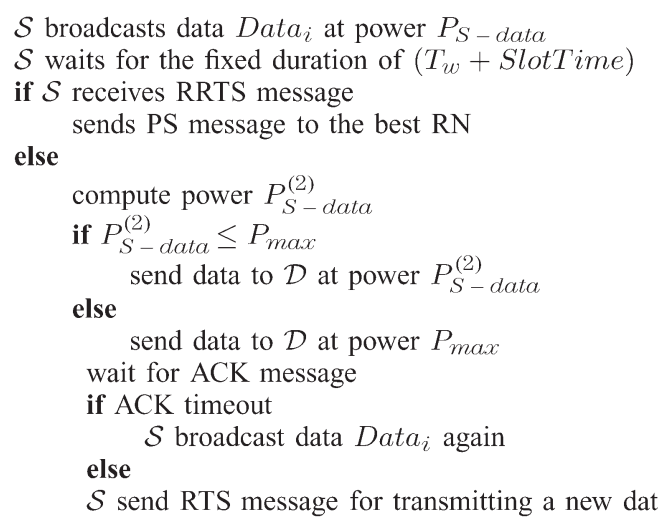

imposed SR data frame to $\mathcal{D}$ at its precalculated transmission 378 power of $P_{\mathcal{R}^{\min }}$ after an SIFS period, acting as the best RN, 379 as shown in Fig. 2. Finally, at the DN, the classic automatic 380 repeat request procedure will be initiated, when receiving the 381 forwarded data and successfully decoding and combing it with 382 the most recent direct transmission during Step I of Phase II. 383

2) Source Retransmission: If none of the RNs competes for 384 a transmission opportunity or multiple RRTS messages collided 385 at the $\mathrm{SN}, \mathcal{S}$ directly sends its data to $\mathcal{D}$ as a replica without 386 relaying. This transmission takes place either at the specific 387 transmit power of $P_{\mathcal{S} \text {-data }}^{(2)}$, which is capable of guaranteeing 388 the expected rate of $\alpha C_{\mathcal{S}, \mathcal{D}}^{\max }$, or failing that, it resorts to using 389 the maximum affordable transmit power of $P_{\max }$, as shown 390 in Table II. If $\mathcal{D}$ receives this data frame, it replies with an 391 acknowledgment (ACK) message to $\mathcal{S}$ after successfully de- 392 coding and combining the frame with the most recent erroneous 393 data frame broadcast by $\mathcal{S}$. If $\mathcal{S}$ does not receive any response 394 from $\mathcal{D}$ before the timer set for waiting for an ACK message 395 is expired, it will broadcast its data again at power of $P_{\mathcal{S}-\text { data }} 396$ to seek cooperation, and the RN selection procedure described 397 earlier is repeated, as shown in Table II.

\section{Simulation Results}

To evaluate the achievable performance of the proposed 400 scheme, we present our simulation results based on Omnet++. 401 Based on the network model introduced in Section II-A, we 402 consider two scenarios to investigate both the achievable rate 403 and EC improvement, and to analyze the RN's behavior. 404 In the first scenario, all the RNs are randomly distributed across 405 the entire network area, whereas $\mathcal{S}$ and $\mathcal{D}$ have fixed positions. 406 The network size considered ranges from $u=5$ nodes to $u=407$ 30 nodes for the sake of evaluating the influence of the size 408 of the networks on the achievable rate and EC. In the other sce- 409 nario, we consider a small network supporting $u=5$ nodes, i.e., 410 $\mathcal{S}, \mathcal{D}$, and three RNs, where all the nodes have fixed positions. 411 One of the three RNs is located at the position of $d=1 / 4$ along 412 the SD link. Another RN is in the middle of the SD link at 413 $d=1 / 2$, whereas the third $\mathrm{RN}$ is at the point $d=3 / 4$ of the SD 414 link. In the given two scenarios, the values of $P_{\max }$ and $P_{\mathcal{S} \text {-data }} 415$ are 2 and $1 \mathrm{~mW}$, respectively. The size of CWmin is 7, whereas 416 SlotTime is set to $20 \mu \mathrm{s}$. Furthermore, the length of SIFS is 417 


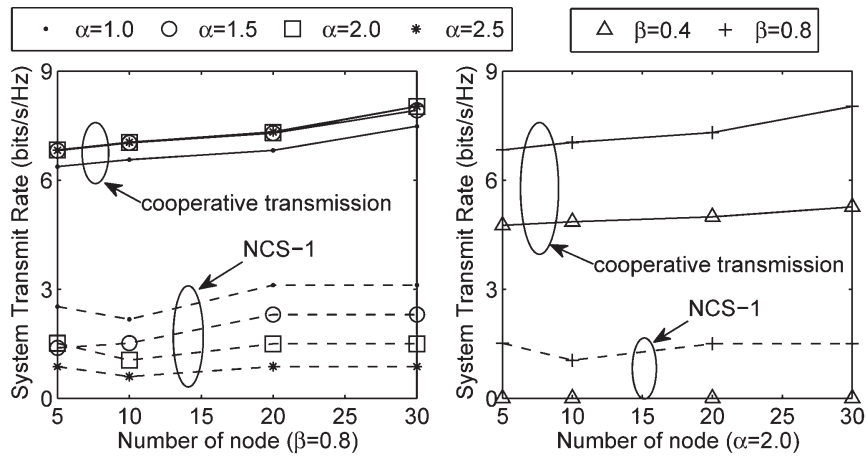

Fig. 4. System's total achievable rate improvement.

$41810 \mu \mathrm{s}$. The length of the data frame generated at the application 419 layer is $1024 \mathrm{~B}$. The length of the RRTS and PS messages is $42020 \mathrm{~B}$ and $14 \mathrm{~B}$, respectively, whereas that of the RTS and $421 \mathrm{CTS}$ is 24 and $18 \mathrm{~B}$. The greedy factor $\alpha$ ranges from 1 to 3 , 422 whereas the value of $\beta$ ranges from 0 to 0.8 . Both $\alpha$ and $\beta$ are 423 predetermined for each simulation.

424 Two noncooperative systems are introduced as the bench425 markers of our comparisons. We compare the system's achiev426 able total transmit rate (TTR) constituted by the sum of the 427 SN's and RN's transmit rate to that of the noncooperative 428 system 1 (NCS-1), which consumes the same total transmission 429 energy as our CSLS (WW-CSLS). Additionally, we compare 430 the total transmission EC to that of the noncooperative system 2 431 (NCS-2), which is capable of achieving the same TTR as our 432 WW-CSLS. Since the SN's data is transmitted twice by itself 433 and additionally by the best RN, if the cooperative transmission 434 is successful, two direct transmission phases are exploited in 435 both NCS- 1 and NCS-2. When aiming for investigating the 436 effect of our relay selection scheme, we compare the achievable 437 performance of our WW-CSLS to that of a random CSLS 438 (Ran-CSLS), where the best RN is randomly selected with439 out considering the transmit power required for providing a 440 successful cooperative transmission. To evaluate their perfor441 mance, we adopt the idealized simplifying assumption that the 442 control messages are received without errors in both NCS-1 443 and NCS-2, as well as in WW-CSLS. In Sections IV-E and F, 444 we investigated a more practical network.

\section{A. Effect of Cooperative Transmission}

446 Let us now investigate the effects of cooperative transmission 447 on the TTR and EC by comparing the performance achieved in 448 the first scenario and NCS-1 and in NCS-2.

449 1) Achievable Transmit Rate: Fig. 4 compares the system's 450 TTR, namely, the sum of both the SN's rate and the RN's rate 451 achieved by the WW-CSLS relying on our WW cooperative 452 MAC protocol to that of NCS-1. It is observed in Fig. 4 that, 453 as expected, the system's achievable TTR relying on our WW454 CSLS is higher than $6 \mathrm{bit} / \mathrm{s} / \mathrm{Hz}$, even for $\alpha=1$ and $\beta=0.8$, 455 which is more than twice as high as that achieved by NCS- 1 , 456 which consumes the same total transmission energy, given the 457 same values of $\alpha$ and $\beta$. Additionally, for $\beta=0.4$ and $\alpha=2$, 458 the system's TTR achieved by our WW-CSLS is in excess of $4594 \mathrm{bit} / \mathrm{s} / \mathrm{Hz}$, while in fact, no successful transmissions may be

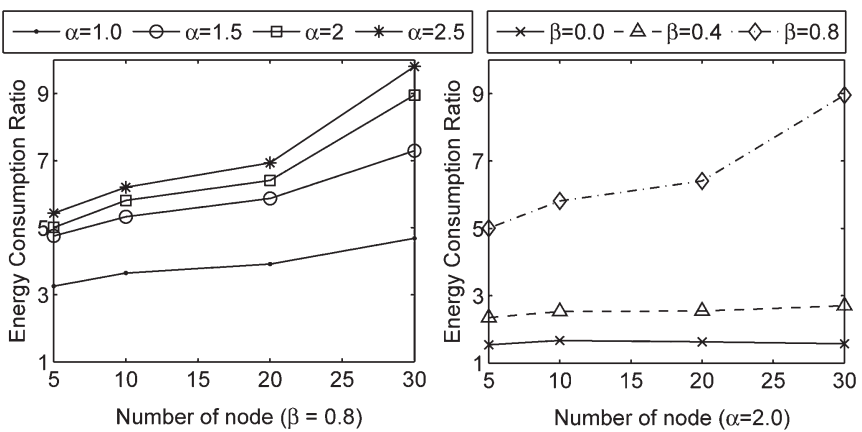

Fig. 5. Energy consumption ratio of $E_{\text {noncoop }} / E_{\text {coop }}$.

supported in NCS-1 for the same values of $\alpha$ and $\beta$ due to 460 the system's low EC. Hence, the proposed WW cooperative 461 MAC protocol is capable of providing a considerable TTR im- 462 provement, despite consuming low energy. As shown in Fig. 4, 463 the system's TTR achieved by our WW-CSLS is increased, 464 when $\mathcal{S}$ becomes greedier due to the SN's increased transmit 465 rate requirement. Additionally, when $\beta$ is increased, the best 466 RN will be rewarded by a considerably higher rate for its own 467 traffic, provided that the cooperation is successful. Hence, the 468 system's TTR is increased, when the RN becomes greedier, 469 as shown in Fig. 4. Moreover, the achievable TTR of our 470 WW-CSLS is gradually increased, when the network becomes 471 larger. The above investigations imply that the proposed WW 472 cooperative MAC protocol is capable of providing significant 473 TTR improvements.

474

2) Energy Consumption: Fig. 5 shows the achievable EC 475 ratio (ECR) of $E_{\text {noncoop }} / E_{\text {coop }}$, where $E_{\text {coop }}$ denotes the sys- 476 tem's total transmission $\mathrm{EC}^{5}$ for our cooperative MAC protocol 477 and $E_{\text {noncoop }}$ represents that of NCS-2, which is capable of 478 achieving the same system's TTR as our WW-CSLS. As shown 479 in Fig. 5, compared with NCS-2, two third of the system's 480 total energy may be saved by exploiting the proposed WW 481 cooperative MAC protocol, given $\beta=0.8$. The EC $E_{\text {coop }}$ of 482 our WW-CSLS is reduced when $\mathcal{S}$ becomes greedier, which 483 can be also characterized by the TTR of NCS-1 in Fig. 4. 484 By contrast, the EC $E_{\text {noncoop }}$ of NCS-2 is slightly increased, 485 when $\mathcal{S}$ becomes greedier due to the slightly increased system 486 rate of WW-CSLS. Hence, the ECR is increased, when $\mathcal{S} 487$ becomes greedier, as shown in Fig. 5. As $\beta$ is increased, the 488 system's ECR is increased from 1.5 to 5 for $\alpha=2$ and $u=5,489$ as shown in Fig. 5. When the RNs become greedier, fewer 490 RNs can afford the increased power required for successfully 491 forwarding the SPC data. However, the transmit rate achieved 492 by the best RN is considerably increased. Hence, an increased 493 total energy is required by NCS-2 for the sake of achieving the 494 same system rate as our WW-CSLS. Therefore, the system's 495 ECR of $E_{\text {noncoop }} / E_{\text {coop }}$ is increased when the RN becomes 496 greedier. Based on the given discussions, the proposed WW co- 497 operative MAC protocol is capable of achieving a considerable 498 system rate improvement while offering a satisfactory energy 499 efficiency.

\footnotetext{
${ }^{5}$ It is reasonable to focus on the transmission EC and ignore the circuit processing EC in a large network where the transmission EC is dominant in the total EC [26].
} 

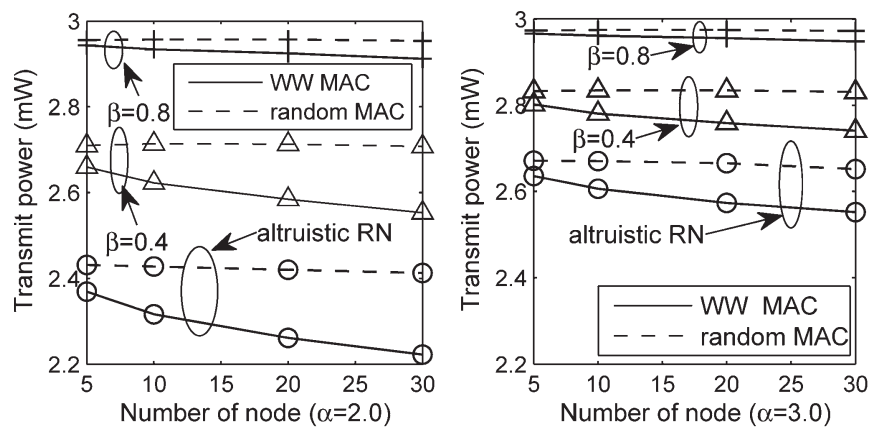

Fig. 6. System data transmit power consumed by our WW-CSLS and Ran-CSLS

\section{B. Effect of Relay Selection}

502 Let us now investigate the effect of the proposed RN selec503 tion scheme by evaluating the achievable performance of our 504 WW-CSLS and Ran-CSLS, where the best RN is randomly 505 selected.

506 1) Transmit Power: According to the proposed WW co507 operative MAC protocol, the specific RN that promises the 508 lowest transmit power $P_{\mathcal{R}_{i}}$ required for successfully conveying 509 superposition-coded data is selected as the best RN. However, 510 the best RN is randomly selected in Ran-CSLS without consid511 ering any system parameters, such as the transmit power $P_{\mathcal{R}_{i}}$. 512 Hence, the RN's transmit power $P_{\mathcal{R}_{i}}$ is the crucial parameter for 513 investigating the effect of the proposed RN selection scheme. 514 Fig. 6 quantifies the system's total data transmit power (TDTP) 515 for our WW-CSLS and that is consumed in Ran-CSLS. The 516 system's TDTP is defined as the sum of the SN's transmit power 517 required for conveying its data plus the RN's transmit power 518 necessitated for delivering the superposition-coded data.

519 Based on the proposed backoff algorithm, the system's TDTP 520 consumed in the WW-CSLS is lower than that of the Ran521 CSLS, as shown in Fig. 6. When the SN or RN becomes greed522 ier, less RNs can afford the increased transmit power required 523 to provide successful cooperative transmission assistance. This 524 phenomenon increases the probability that the same RN is 525 selected as the best RN in both WW-CSLS and Ran-CSLS. 526 Hence, the difference between the TDTP of our WW-CSLS and 527 that of Ran-CSLS is reduced when either $\alpha$ or $\beta$ is increased, 528 as shown in Fig. 6. Moreover, the TDTP of both WW-CSLS 529 and of the Ran-CSLS is reduced when the network hosts more 530 RNs due to the increased probability of having RNs, which 531 promise to reduce the transmit power in comparison with a 532 smaller network. However, the probability of the event that a 533 low-quality RN, namely, one which requires a higher transmit 534 power than other RNs, is selected as the best RN in the Ran535 CSLS is increased, when the network becomes larger. Hence, 536 compared with Ran-CSLS, an increased TDTP is saved by our 537 WW-CSLS when the network's size is increased.

538 2) Achievable Transmit Rate: Fig. 7 compares the system's 539 TTR, namely, the sum of both the SN's rate and the RN's rate 540 achieved by our WW-CSLS to that achieved by Ran-CSLS. 541 As shown in Fig. 7, the system's achievable TTR relying on $542 \mathrm{WW}-\mathrm{CSLS}$ is $8 \mathrm{bit} / \mathrm{s} / \mathrm{Hz}$ for $\beta=0.8$ and $u=30$, whereas a 543 lower TTR of $6.5 \mathrm{bit} / \mathrm{s} / \mathrm{Hz}$ is achieved by Ran-CSLS, given $\beta$ 544 and the network size. Compared with Ran-CSLS, the system's

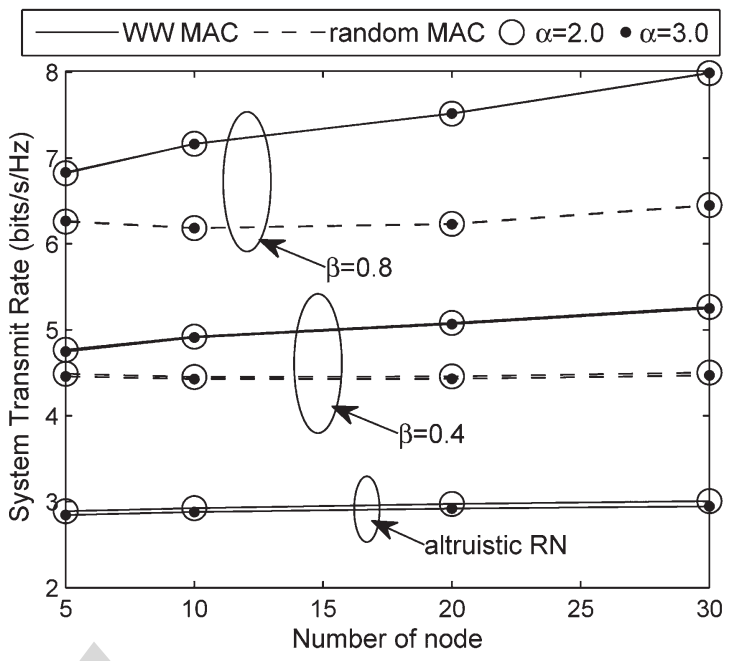

Fig. 7. System's total achievable rate improvement of our WW-CSLS and Ran-CSLS.

TTR can be improved by our WW-CSLS, even for lower $\beta 545$ values and for smaller networks, e.g., for $\beta=0.4$ and $u=5,546$ as shown in Fig. 7. Based on WW-CSLS, the specific RN that 547 promises lower transmit power of $P_{\mathcal{R}_{i}}$ may achieve a higher 548 transmit rate of $\beta C_{\mathcal{R}_{i}, \mathcal{D}}^{\max }$ due to having an improved RD link. 549 Hence, compared with Ran-CSLS, a higher TTR is achieved 550 by our WW-CSLS relying on selecting the specific RN, which 551 promises the lowest transmit power $P_{\mathcal{R}_{i}}$. 552

Observe in Fig. 7 that the proposed WW cooperative MAC 553 protocol is capable of providing a higher TTR improvement 554 than Ran-CSLS, when $\beta$ is increased. When an RN be- 555 comes greedier, its target transmit rate is increased. This phe- 556 nomenon increases the difference between the RN's transmit 557 rate achieved by WW-CSLS and that achieved by Ran-CSLS 558 when the RN that suffers from a low-quality RD link is selected 559 by Ran-CSLS. Hence, the difference between the TTR of WW- 560 CSLS and that of Ran-CSLS is increased when the RN becomes 561 greedier. Considering the CSLS, where the RN altruistically 562 forwards data for $\mathcal{S}$, the system's TTR is equal to the SN's rate. 563 Hence, the system's TTR remains the same, regardless of which 564 particular candidate RN is selected as the best RN when the 565 RNs are altruistic, as shown in Fig. 7.

566

As shown in Fig. 7, the system's TTR achieved by our WW- 567 CSLS is increased, when the network becomes larger. However, 568 the effect of the network's size on the TTR achieved by Ran- 569 CSLS is not as obvious as that on our WW-CSLS. When the 570 network hosts more RNs, the number of candidate RNs may 571 be increased. This phenomenon increases the probability that 572 a low-quality $\mathrm{RN}$ having a lower transmit rate is selected as 573 the best RN in Ran-CSLS. However, these low-quality RNs 574 cannot win the cooperative transmission opportunity in our 575 WW-CSLS if the specific RN promising a reduced transmit 576 power also contends for the transmission opportunity. Hence, 577 a higher TTR improvement is provided by the proposed WW 578 cooperative MAC protocol, as the network becomes larger, 579 as shown in Fig. 7. The given investigations imply that the 580 proposed WW cooperative MAC protocol is capable of saving 581 a substantial amount of transmit power while simultaneously 582 


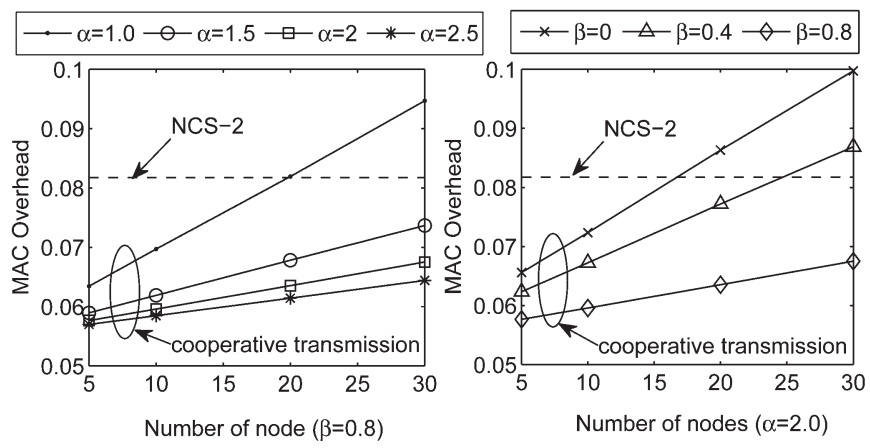

Fig. 8. MAC overhead for $\beta=0.8$ or $\alpha=2$.

583 providing significant TTR improvements compared with 584 Ran-CSLS.

\section{C. MAC Overhead}

586 Fig. 8 compares the MAC overhead of the proposed coop587 erative MAC protocol with that of NCS-2, which is based on 588 the RTS/CTS signaling regime of the IEEE 802.11 standards 589 [22]. The MAC overhead is defined as the ratio of $\left(\mathcal{N}_{\text {mac }-c}+\right.$ $\left.590 \mathcal{N}_{\text {mac }-h}+\mathcal{N}_{\text {mac }-t}\right) / \mathcal{N}_{\text {mac }-d}$, where $\mathcal{N}_{\text {mac }-c}$ denotes the num591 ber of bits of all MAC control messages, and $\mathcal{N}_{\text {mac }-h}$ and $592 \mathcal{N}_{\text {mac }-t}$ represent the number of header and tailing bits of the 593 MAC data frame, respectively. Furthermore, $\mathcal{N}_{\text {mac }-d}$ denotes 594 the number of bits in the payload data packet, including the 595 headers introduced by the higher layers. Observe in Fig. 8 that 596 the MAC overhead of the proposed WW cooperative MAC 597 protocol decreases, when either $\alpha$ or $\beta$ increases, because the 598 number of candidate RNs is reduced, whereas the $\mathrm{SN}$ or the $599 \mathrm{RN}$ becomes greedier. Compared with the traditional RTS/CTS 600 scheme specified in the IEEE 802.11 standards [22], the RRTS 601 message and the PS message are introduced into our WW-CSLS 602 to assist with RN selection if cooperation can be exploited. 603 However, compared with NCS-2, the RN's data can be also 604 transmitted with the aid of cooperation in WW-CSLS. Since 605 the length of the RN's data frames is higher than that of the 606 extra control messages, the MAC overhead introduced by our $607 \mathrm{WW}$ protocol is lower than that of the NCS-2 when the network 608 size is smaller than $u=20$. Although the overhead of our 609 WW-CSLS becomes higher than that of NCS-2 when the 610 network hosts more than $u=20$ nodes, the MAC overhead 611 introduced by our WW protocol always remains lower than 6120.1 for $\beta=0.8$ or $\alpha=2$.

\section{D. Relay Behavior}

614 To investigate the behavior of the relays, we analyze both the 615 transmission probability and the achievable rate improvement 616 of each RN for the configuration of $\alpha=2$ in the network 617 hosting $u=5$ nodes, as shown in Fig. 9(a) and (b). Upon 618 increasing $\beta$, the transmission probability of the RNs at " $d=$ $6191 / 4$ " and " $d=1 / 2$ " decreases, whereas that of the RN at 620 " $d=3 / 4$ " increases, as shown in Fig. 9(a). The $\mathrm{RN}$ at " $d=$ 621 3/4" always benefits from the highest transmission probability, 622 whereas the $\mathrm{RN}$ at " $d=1 / 4$ " has the lowest probability of 623 cooperative opportunities. As a benefit of its highest transmis624 sion probability, the $\mathrm{RN}$ at " $d=3 / 4$ " maintains the highest

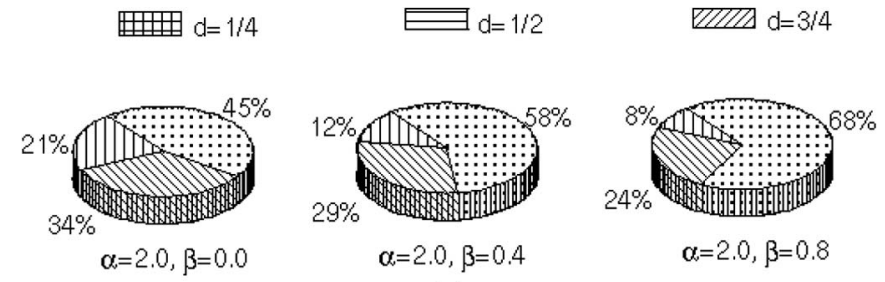

(a)

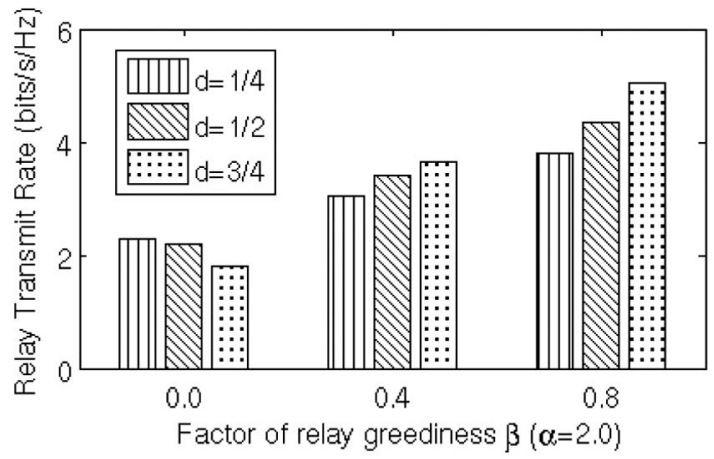

(b)

Fig. 9. RN transmission probability and the achievable rate improvement in a network hosting $u=5$ nodes, namely, $\mathcal{S}, \mathcal{D}$, and three RNs. (a) Relay transmission probability. (b) Relay achievable rate.

achievable rate improvement, which is above $5 \mathrm{bits} / \mathrm{s} / \mathrm{Hz}$ for 625 $\beta=0.8$ and $\alpha=2$. The achievable $\mathrm{RN}$-rate improvement at 626 " $d=1 / 4$ " is lower than that of the $\mathrm{RN}$ at " $d=1 / 2$," as shown in 627 Fig. 9(b). However, when the three RNs altruistically dedicate 628 themselves solely to forwarding data frames for $\mathcal{S}(\beta=0)$, the 629 achievable RN-rate improvement at " $d=1 / 4$ " is higher than 630 that of the other relays. Naturally, if the RNs become selfish, 631 their improved transmission probability leads to an increased 632 total throughput.

\section{E. Effect of Erroneous RTS Message}

The contention caused by hidden SNs or RNs may corrupt 635 the transmission of data and control messages. Apart from the 636 effects of corrupted RTS messages, the erroneous transmission 637 of both other control messages and of data have been considered 638 in our WW cooperative MAC protocol. Hence, the effect of 639 corrupted RTS messages on the system's transmit rate and on 640 the ECR of $E_{\text {rts-error }} / E_{\text {error-free }}$ that are achieved by our 641 WW-CSLS are evaluated, as shown in Fig. 10(a) and (b). The 642 variable $E_{\text {rts-error }}$ denotes the system's total EC for WW- 643 CSLS, where the RTS message may be corrupted. Furthermore, 644 $E_{\text {error-free }}$ is the system's total EC for WW-CSLS, where 645 error-free control messages are assumed. It is observed in 646 Fig. 10(a) and (b) that, when the RTS error probability is 647 increased, the system's TTR is decreased, and an increased 648 total system energy is dissipated by our WW-CSLS because 649 having more potentially erroneous RTS transmissions reduces 650 the probability of successful transmission, and the extra RTS 651 message retransmissions consume extra energy.

\section{F. Effect of Imperfect Channel Estimation}

To evaluate the overall system performance of our WW 654 cooperative protocol in a more practical scenario, we now 655 


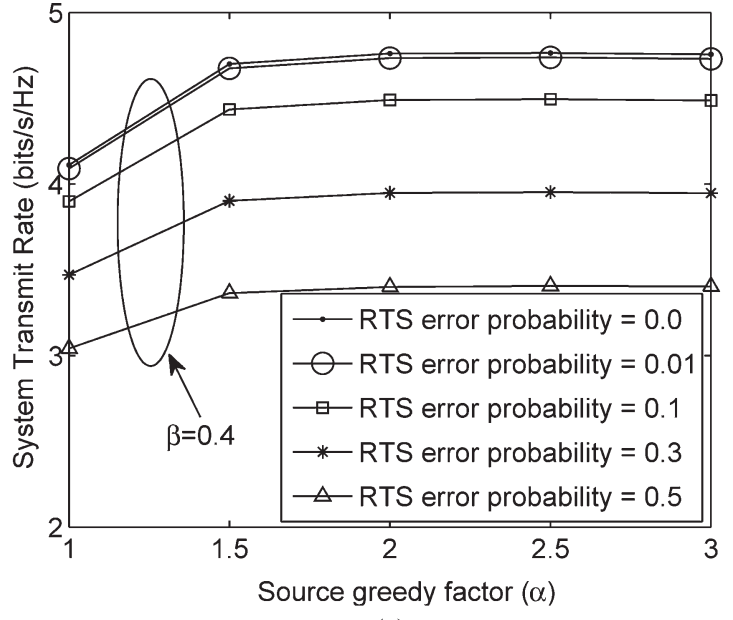

(a)

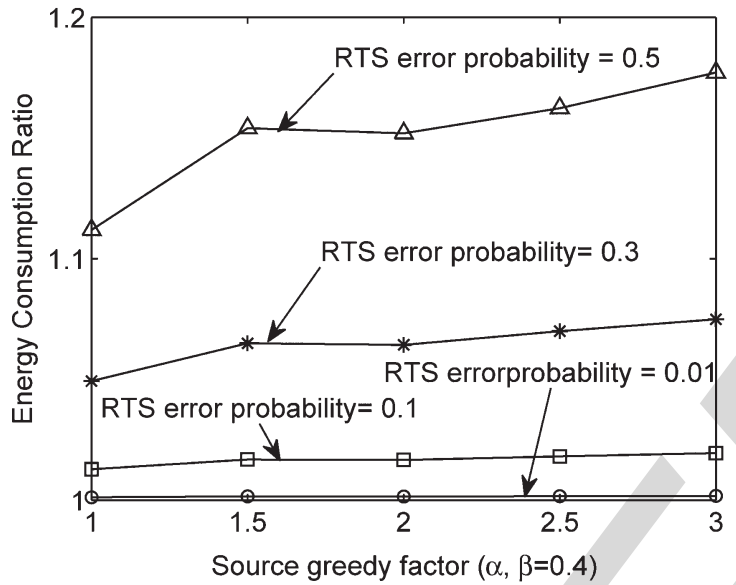

(b)

Fig. 10. System's total achievable transmit rate and system's ECR of $E_{\text {rts-error }} / E_{\text {error-free }}$ versus the SN's greedy factor parameterized with different RTS message error probabilities. (a) System's TTR. (b) System's ECR of $E_{\text {rts-error }} / E_{\text {error-free. }}$.

656 introduce Gaussion-distributed CSI estimation errors into our 657 WW-CSLS, instead of relying on the idealized simplifying 658 assumption of perfect CSI. The normalized mean square error 659 (NMSE) of the Gaussian channel estimation errors was defined 660 as $10 \log \left(E\left\{\|h-\hat{h}\|^{2}\right\} / E\left\{\|h\|^{2}\right\}\right)$ in decibels [27]. Compared 661 with the performance achieved by assuming perfect CSI, the 662 realistic imperfect channel estimation reduces the system's 663 attainable transmit rate and dramatically increases the system's 664 ECR of $E_{\text {error }} / E_{\text {perfect }}$, as shown in Fig. 11(a) and (b), respec665 tively. Variable $E_{\text {error }}$ denotes the system's energy consumed 666 by the CSLS relying on realistic imperfect channel estimation, 667 whereas $E_{\text {perfect }}$ denotes when perfect CSI is assumed. Based 668 on the given discussions, it is necessary to develop a more 669 robust cooperative MAC protocol to reduce the impact of 670 realistic imperfect channel estimation.

\section{G. Effect of Either Superposition Coding or Frame Combining}

672 To evaluate the achievable TTR improvement jointly attained 673 by SPC and SIC, we compare the system's TTR achieved by 674 our WW-CSLS with that of the cooperative system operating 675 without exploiting these techniques, as shown in Fig. 12. Since 676 there are two data frames jointly conveyed by the RN to

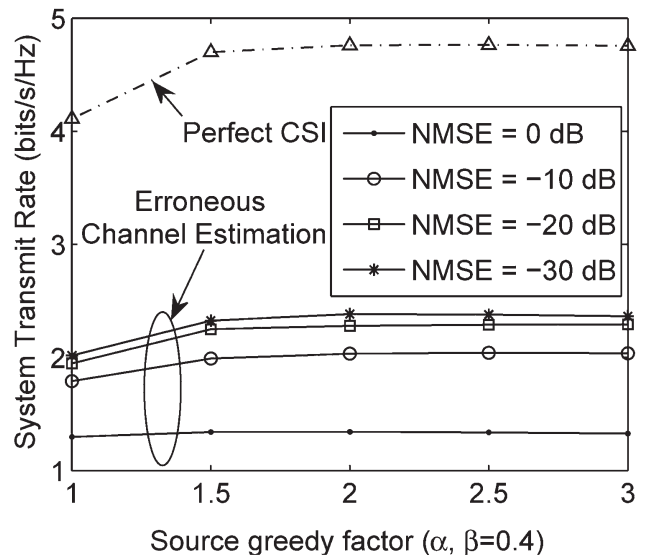

(a)

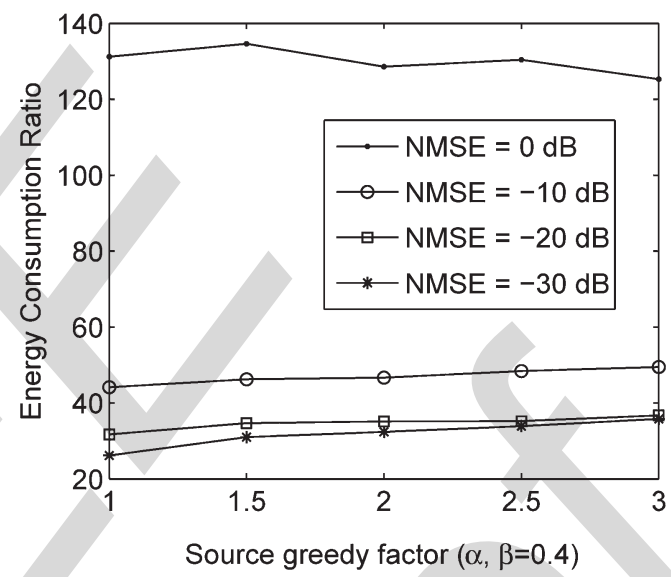

(b)

Fig. 11. System's total achievable transmit rate and system's ECR of $E_{\mathrm{rts}-\text { error }} / E_{\text {error-free }}$ versus the SN's greedy factor parameterized with different channel estimation NMSEs when $\beta=0.4$. (a) System's TTR. (b) System's ECR of $E_{\text {rts-error }} / E_{\text {error-free }}$.

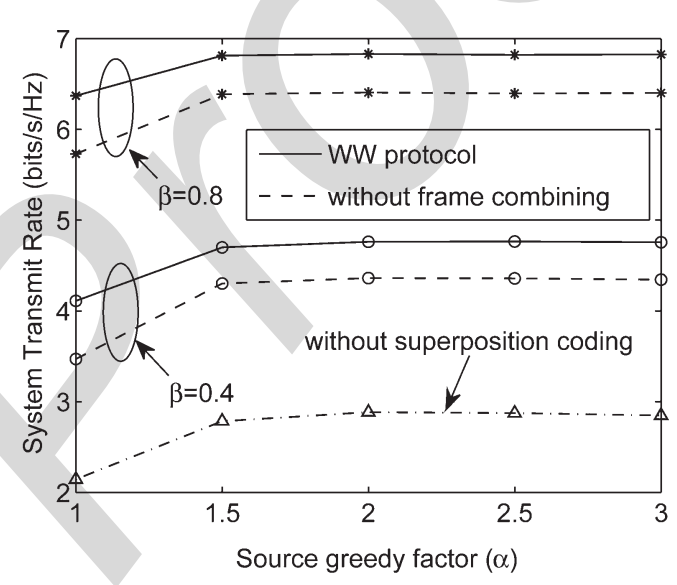

Fig. 12. System's total achievable transmit rate versus the SN's greedy factor both with and without SPC and SIC and frame combining.

$\mathcal{D}$ in our WW-CSLS, the best RN, which does not exploit 677 SPC, is assumed to forward only the SN's data instead of the 678 SPC data. As shown in Fig. 12, the system's TTR may be 679 increased from 2.9 to $6.9 \mathrm{bits} / \mathrm{s} / \mathrm{Hz}$ for $\alpha=2$ and $\beta=0.8$ by 680 jointly exploiting the SPC and SIC. Hence, these techniques are 681 capable of significantly improving the system's transmit rate. 682 To improve the SN's transmit rate, $\mathcal{D}$ invokes frame combining 683 
684 for amalgamating both the direct and relayed SN data after 685 successfully separating the SN's and RN's data. Fig. 12 shows 686 the system's TTR improvement achieved by exploiting frame 687 combining.

\section{CONClusion}

689 In this paper, we have formulated a distributed WW cooper690 ative framework for striking a tradeoff between the achievable 691 system rate improvement and EC and for granting transmission 692 opportunities for the unlicensed RNs. Furthermore, a WW 693 cooperative MAC layer protocol was proposed for implement694 ing our DWWCF. When compared with the corresponding 695 noncooperative system, the proposed scheme is capable of 696 providing a considerable transmit rate and transmission EC 697 improvements. This was achieved with the aid of joint SPC at 698 the RN for both the SN's and RN's data and by combining the 699 SD and RD signals at the DN. Our future work will consider 700 similar interference-limited scenarios relying on a more robust 701 cooperative MAC design.

702

703

704

705

706

707

708

709

710

711

712

713

714

715

716

717

718

719

720

721

722

723

724

725

726

727

728

729

730

731

732

733

734

735

736

\section{REFERENCES}

[1] L. Hanzo, Y. Akhtman, L. Wang, and M. Jiang, MIMO-OFDM for LTE, WIFI and WIMAX: Coherent versus Non-Coherent and Cooperative Turbo-Transceivers. New York, NY, USA: Wiley, 2010.

2] F. Verde, T. Korakis, E. Erkip, and A. Scaglione, "A simple recruitment scheme of multiple nodes for cooperative MAC," IEEE Trans. Commun., vol. 58, no. 9, pp. 2667-2682, Sep. 2010.

[3] G. N. Shirazi, P. Y. Kong, and C. K. Tham, "Distributed reinforcement learning frameworks for cooperative retransmission in wireless networks," IEEE Trans. Veh. Technol., vol. 59, no. 8, pp. 4157-4162, Oct. 2010.

4] T. Luo, V. Srinivasan, and M. Motani, "A metric for DISH networks: Analysis, implications, and applications," IEEE Trans. Mobile Comput., vol. 9, no. 3, pp. 376-389, Mar. 2010.

[5] A. Argyriou, "Cross-layer and cooperative opportunistic network coding in wireless ad hoc networks," IEEE Trans. Veh. Technol., vol. 59, no. 2, pp. 803-812, Feb. 2010

6] H. Shan, W. Zhuang, and Z. Wang, "Distributed cooperative MAC for multihop wireless networks," IEEE Commun. Mag., vol. 47, no. 2, pp. 126-133, Feb. 2009.

7] P. Liu, Z. Tao, S. Narayanan, T. Korakis, and S. S. Panwar, "CoopMAC: A cooperative MAC for wireless LANs," IEEE J. Sel. Areas Commun., vol. 25, no. 2, pp. 340-354, Feb. 2007.

[8] J. Feng, R. Zhong, S. X. Ng, and L. Hanzo, "Relay selection for energy-efficient cooperative media access control," in Proc. IEEE WCNC, Cancun, Mexico, Mar. 2011, pp. 287-292.

9] Z. Zhou, S. Zhou, J. Cui, and S. Cui, "Energy-efficient cooperative communication based on power control and selective single-relay in wireless sensor networks," IEEE Trans. Commun., vol. 7, no. 8, pp. 3066-3078, Aug. 2008.

10] S. Mehta and K. S. Kwak, "An energy-efficient MAC protocol in wireless sensor networks: A game theoretic approach," EURASIP J. Wireless Commun. Netw., vol. 2010, pp. 1-10, May 2010.

1] K. B. Letaief and W. Zhang, "Cooperative communications for cognitive radio networks," Proc. IEEE, vol. 97, no. 5, pp. 878-893, May 2009.

2] R. Madan, N. B. Mehta, A. F. Molisch, and J. Zhang, "Energy-efficient cooperative relaying over fading channels with simple relay selection," IEEE Trans. Commun., vol. 7, no. 8, pp. 3013-3025, Aug. 2008.

3] T. Luo, M. Motani, and V. Srinivasan, "Energy-efficient strategies for cooperative multi-channel MAC protocols," IEEE Trans. Mobile Comput., vol. 11, no. 4, pp. 553-566, Apr. 2012.

14] Y. Zhou, J. Liu, C. Zhai, and L. Zheng, "Two-transmitter two-receiver cooperative MAC protocol: Cross-layer design and performance analysis," IEEE Trans. Veh. Technol., vol. 59, no. 8, pp. 4116-4127, Oct. 2010.

5] Q. Zhao and B. Sadler, "A survey of dynamic spectrum access," IEEE Signal Proc. Mag., vol. 24, pp. 79-89, May 2007.

6] A. Mukherjee and H. M. Kwon, "General auction-theoretic strategies for distributed partner selection in cooperative wireless networks," IEEE Trans. Commun., vol. 58, no. 10, pp. 2903-2915, Oct. 2010.
[17] I. Stanojev, O. Simeone, U. Spagnolini, Y. Bar-Ness, and R. Pickholtz, 751 "Cooperative ARQ via auction-based spectrum leasing," IEEE Trans. 752 Commun., vol. 58, no. 6, pp. 1843-1856, Jun. 2010.

[18] F. Pantisano, M. Bennis, W. Saad, and M. Debbah, "Spectrum leasing as 754 an incentive towards uplink macrocell and femtocell cooperation," IEEE 755 J. Sel. Areas Commun., vol. 30, no. 3, pp. 617-630, Apr. 2012.

[19] Asaduzzaman, H. Y. Kong, and I. Koo, "Opportunistic relaying based 757 spectrum leasing for cognitive radio networks," J. Commun. Netw., 758 vol. 13, no. 1, pp. 50-55, Feb. 2011.

[20] M. Hafeez and J. Elmirghani, "Analysis of dynamic spectrum leasing 760 for coded bi-directional communication," IEEE J. Sel. Areas Commun., 761 vol. 30, no. 8, pp. 1500-1512, Sep. 2012.

762

[21] S. Jayaweera, M. Bkassiny, and A. Very, "Asymmetric cooperative com- 763 munications based spectrum leasing via auctions in cognitive radio net- 764 works," IEEE Trans. Wireless Commun., vol. 10, no. 8, pp. 2716-2724, 765 Aug. 2011.

[22] IEEE Standard for Information Technology-Telecommunications and 767 Information Exchange Between Systems-Local and Metropolitan Area 768 Networks-Specific Requirements-Part 11: Wireless LAN Medium Access 769 Control (MAC) and Physical Layer (PHY) Specifications, IEEE Std. 770 802.11 , 2007. [Online]. Available: http://standards.ieee.org/getieee802/ 771 802.11.html

[23] D. Chase, "Digital signal design concepts for a time-varying Rician 773 channel," IEEE Trans. Commun., vol. COMM-24, no. 2, pp. 164-172, 774 Feb. 1976.

[24] I. Stanojev, O. Simeone, Y. Bar-ness, and C. You, "Performance of multi- 776 relay collaborative hybrid-ARQ protocols over fading channels," IEEE 777 Commun. Lett., vol. 10, no. 7, pp. 522-524, Jul. 2006.

[25] L. Hanzo, M. Munster, B. Choi, and T. Keller, OFDM and MC-CDMA 779 for Broadcasting Multi-User Communications, WLANs and Broadcasting. 780 Chichester, U.K.: Wiley, 2003.

[26] J. G. S. Cui and A. Bahai, "Energy-constrained modulation optimization," 782 IEEE Trans. Commun., vol. 4, no. 5, pp. 2349-2360, Sep. 2005.

[27] X. Gao, B. Jiang, Z. P. X. You, Y. Xue, and E. Schulz, "Efficient channel 784 estimation for MIMO single-carrier block transmission with dual cyclic 785 timeslot structure," IEEE Trans. Commun., vol. 55, no. 11, pp. 2210- 786 2223, Nov. 2007.

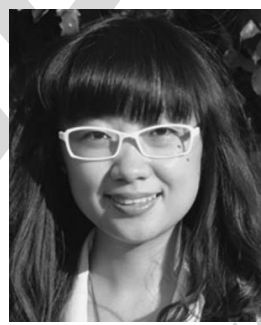

Jiao Feng received the B.Eng. degree and the M.Sc. 788 degree in communications engineering from Jilin 789 University, Jilin, China, in 2007 and 2009, respec- 790 tively. She is currently working toward the Ph.D. 791 degree in wireless communications with the School 792 of Electronics and Computer Science, University of 793 Southampton, Southampton, U.K., under the sup- 794 port of the China-U.K. Excellence Scholarship from 795 both the University of Southampton and the China 796 Scholarship Council.

797

Her research interests include cooperative com- 798 munication, medium-access-control-layer protocols, and cognitive radio 799 networks.

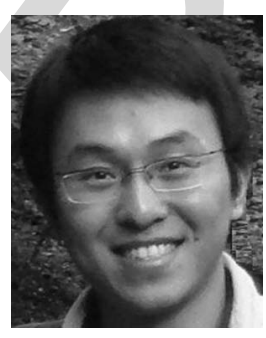

Rong Zhang (M'09) received the B.Sc. degree 801 from Southeast University, Nanjing, China, in 2003802 and the Ph.D. degree from the University of 803 Southampton, Southampton, U.K., in 2009.

From August 2003 to July 2004, he was an En- 805 gineer with China Telecom, Beijing, China. From 806 January 2006 to May 2009, he was a Research As- 807 sistant with the Mobile Virtual Center of Excellence, 808 Hampshire, U.K. From August 2009 to July 2012, he 809 was a Postdoctoral Researcher with the University of 810 Southampton. From August 2012 to January 2013, 811 he took his industrial consulting leave for Huawei Sweden Research and 812 Development as a System Algorithms Specialist. Since February 2013, he has 813 been a Lecturer with the Communications, Signal Processing, and Control 814 Group, Electronics and Computer Science, University of Southampton. He is 815 also a Visiting Researcher with Nanjing University, Nanjing, China, under the 816 Worldwide University Network. He is the author several papers in more than 817 25 journals in prestigious publication avenues [e.g., the IEEE and The Optical 818 Society] and in major conference proceedings.

Dr. Zhang regularly serves as a Reviewer for IEEE TRANSACTIONS/ 820 JouRnAls, and he has been a Technical Program Committeee Member/Invited 821 Session Chair of major conferences several times. He received joint funding 822 from the MVCE and the Engineering and Physical Sciences Research Council. 823 


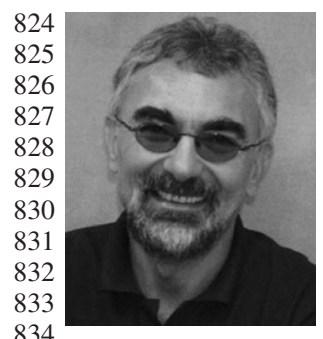

Lajos Hanzo (M'91-SM'92-F'04) received the M.S. degree (with first-class honors) in electronics and the Ph.D. degree from the Technical University of Budapest, Budapest, Hungary, in 1976 and 1983, respectively, the D.Sc. degree from the University of Southampton, Southampton, U.K., in 2004, and the "Doctor Honoris Causa" degree from the Technical University of Budapest in 2009.

During his 35-year career in telecommunications, he has held various research and academic posts in Hungary, Germany, and the U.K. Since 1986, he has 835 been with the School of Electronics and Computer Science, University of 836 Southampton, Southampton, U.K., where he holds the Chair for Telecommuni837 cations. Since 2009, he has been a Chaired Professor with Tsinghua University, 838 Beijing China. He is currently directing a 100-strong academic research team, 839 working on a range of research projects in the field of wireless multimedia 840 communications sponsored by industry; the Engineering and Physical Sciences 841 Research Council, U.K.; the European IST Programme; and the Mobile Virtual 842 Centre of Excellence, U.K. He is an enthusiastic supporter of industrial and 843 academic liaison and offers a range of industrial courses. He has successfully 844 supervised 80 Ph.D. students, coauthored 20 John Wiley/IEEE Press books on 845 mobile radio communications totaling in excess of 10000 pages, published 846 more than 1250 research entries on IEEE Xplore, and presented keynote 847 lectures. (For further information on research in progress and associated 848 publications, please refer to http://www-mobile.ecs.soton.ac.uk.)

849 Dr. Hanzo is Fellow of the Royal Academy of Engineering, U.K., a Fellow 850 of the Institution of Electrical Engineers, and a Governor of the IEEE Vehicular 851 Technology Society. He has been a Technical Program Committee Chair and a 852 General Chair for IEEE conferences. During 2008-2012, he was the Editor-in853 Chief of the IEEE Press. He has received a number of distinctions.

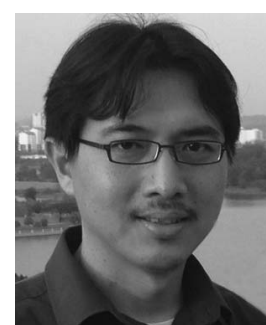

Soon Xin Ng (S'99-M'03-SM'08) received the 854 B.Eng. degree (first-class honors) in electronics en- 855 gineering and the Ph.D. degree in wireless com- 856 munications from the University of Southampton, 857 Southampton, U.K., in 1999 and 2002, respectively. 858 From 2003 to 2006, he was a Postdoctoral Re- 859 search Fellow working on collaborative European 860 research projects known as SCOUT, NEWCOM, 861 and PHOENIX. Since August 2006, he has been a 862 member of the academic staff with Electronics and 863 Computer Science, University of Southampton. He 864 is involved in the OPTIMIX and CONCERTO European projects and the IU- 865 ATC and UC4G projects. He is currently a Senior Lecturer with the University 866 of Southampton. He is the author of over 150 papers and a coauthor of two 867 John Wiley/IEEE Press books in his field of expertise. His research inter- 868 ests include adaptive coded modulation, coded modulation, channel coding, 869 space-time coding, joint source and channel coding, iterative detection, orthog- 870 onal frequency-division multiplexing, multiple-input-multiple-output systems, 871 cooperative communications, distributed coding, quantum error correction 872 codes, and joint wireless-and-optical-fiber communications.

Dr. $\mathrm{Ng}$ is a Chartered Engineer and a Fellow of the Higher Education 874 Academy in the U.K. 


\section{AUTHOR QUERIES}

\section{AUTHOR PLEASE ANSWER ALL QUERIES}

AQ1 = Please provide keywords.

AQ2 = Please provide expanded form of OF.

AQ3 = What is the first initial of author Asaduzzaman?

\section{END OF ALL QUERIES}




\title{
Cooperative Medium Access Control Based on Spectrum Leasing
}

\author{
3 Jiao Feng, Rong Zhang, Member, IEEE, Lajos Hanzo, Fellow, IEEE, and Soon Xin Ng, Senior Member, IEEE
}

\begin{abstract}
4 Abstract-Based on cooperative spectrum leasing, a distributed 5 "win-win" (WW) cooperative framework is designed to encourage 6 the licensed source node (SN) to lease some part of its spectral 7 resources to the unlicensed relay node $(\mathrm{RN})$ for the sake of si8 multaneously improving the SN's achievable rate and for reducing 9 the energy consumption (EC). The potential candidate RNs carry 10 out autonomous decisions concerning whether to contend for a 11 cooperative transmission opportunity, which could dissipate some 12 of their battery power, while conveying their traffic in light of their 13 individual service requirements. Furthermore, a WW cooperative 14 medium-access-control (MAC) protocol is designed to implement 15 the proposed distributed WW cooperative framework. Simulation 16 results demonstrate that our WW cooperative MAC protocol is 17 capable of providing both substantial rate improvements and 18 considerable energy savings for the cooperative spectrum leasing 19 system.
\end{abstract}

20 Index Terms-Author, please supply index terms/keywords 21 for your paper. To download the IEEE Taxonomy go to 22 http://www.ieee.org/documents/taxonomy_v101.pdf.

\section{INTRODUCTION}

24 OOPERATIVE communications techniques have recently 25 attracted substantial research attention [1] as a benefit 26 of their significant throughput improvements, energy savings, 27 and coverage enhancements. However, these benefits may be 28 eroded by the conventional higher layer protocols, which were 29 designed for classic noncooperative systems. Hence, it is im30 portant to design appropriate medium-access-control (MAC) 31 protocols to support cooperative physical layer techniques.

32 In contrast with the legacy wireless MAC protocols, cooper33 ative MAC protocols aim to cooperatively schedule the medium 34 access of all nodes while allowing the relay nodes (RNs) to 35 buffer and forward the others' data frames using the broadcast 36 nature of the wireless network, instead of ignoring these data 37 frames. There are numerous contributions in the literature on 38 designing cooperative MAC protocols, most of which aim to

Manuscript received December 11, 2012; revised April 28, 2013; accepted July 4, 2013. This work was supported in part by the Research Councils UK under the auspices of the U.K.-India Advanced Technology Center in Wireless Communications, the European Union CONCERTO Project, and the European Research Council's Advanced Fellow Grant and in part by the Royal Society's Wolfson Research Merit Award. The review of this paper was coordinated by Prof. J. Tang.

The authors are with the School of Electronics and Computer Science, University of Southampton, Southampton SO17 1BJ, U.K. (e-mail: 1h@ecs. soton.ac.uk).

Color versions of one or more of the figures in this paper are available online at http://ieeexplore.ieee.org.

Digital Object Identifier 10.1109/TVT.2013.2272895 maximize the throughput [2]-[6], including the widely recog- 39 nized CoopMAC of [7]. However, a potential impediment of the 40 CoopMAC is that its energy efficiency was traded off against 41 the throughput benefits claimed. Therefore, [8]-[12] aimed to 42 minimize the energy consumption (EC) by developing energy- 43 efficient cooperative MAC protocols. To jointly consider these 44 conflicting design objectives, Luo et al. [13] and Zhou et al. 45 [14] designed meritorious algorithms to improve the achievable 46 throughput and to simultaneously enhance the energy efficiency 47 achieved.

However, the aforementioned cooperative MAC protocols, 49 such as CoopMAC, were developed based on the common 50 assumption that the relays agree to altruistically forward the 51 data of the source node (SN). This unconditional altruistic 52 behavior is unrealistic to expect from mobile stations. In fact, 53 a greedy $\mathrm{RN}$ behavior is likely to be the norm in spectrum 54 leasing [15], where the licensed SN intends to lease some part 55 of its spectral resources to the unlicensed $\mathrm{RN}$ in exchange for 56 appropriate "remuneration." In this spectrum leasing system, 57 the unlicensed RNs also have an incentive to support the SN 58 to achieve its quality-of-service (QoS) target in exchange for a 59 transmission opportunity. This cooperation allows both the SN 60 and the RN to satisfy its individual requirement. Based on this 61 cooperative spectrum leasing system, some early theoretical 62 studies have been conducted in [16]-[21]. Bearing in mind the 63 greedy behavior of the mobile RNs, meritorious game-theoretic 64 frameworks were proposed in [16]-[19] to maximize the SN's 65 transmit rate while simultaneously satisfying the requirements 66 of the RNs. Based on game theory, Hafeez and Elmirghani 67 [20] and Jayaweera et al. [21] aimed to minimize the $E C$ of 68 cooperative spectrum leasing systems by designing beneficial 69 game-aided strategies. However, the joint optimization of the 70 transmit rate and of the $\mathrm{EC}$ has not been considered in these 71 existing works. Furthermore, the design of an appropriate 72 cooperative MAC protocol for practically implementing the 73 theoretical framework was not discussed in [16]-[21]. 74

Against this backdrop, the contributions of this paper are as 75 follows.

1) We first formulate a distributed "win-win" (WW) coop- 77 erative framework (DWWCF) to encourage the SN to 78 lease part of its spectral resources to the unlicensed RN 79 for the sake of improving the SN's transmit rate and for 80 simultaneously reducing the SN's $E C$ while ensuring that 81 the unlicensed RNs are capable of securing a transmission 82 opportunity for their own traffic and for satisfying their 83 QoS. Furthermore, the proposed DWWCF selects the 84 

121 network's architecture and our DWWCF are introduced in 122 Section II. Section III describes the proposed WW cooperative 123 MAC protocol, whereas in Section IV, the attainable perfor124 mance of our scheme is quantified. Finally, we conclude in 125 Section V.

\section{System Model ANd Distributed Win-Win COOPERATIVE FRAMEWORK}

\section{A. System Model}

129 Before embarking on outlining our DWWCF, we introduce 130 our network topology and outline our assumptions.

131 As shown in Fig. 1, we consider a cooperative network 132 having a single $\mathrm{SN} \mathcal{S}$ and a total of $N$ RNs in the set $\mathcal{R}=$ $133\left\{\mathcal{R}_{1}, \ldots, \mathcal{R}_{N}\right\}$, as well as a common DN $\mathcal{D}$, where $\mathcal{D}$ may be 134 a base station (BS) or an ad hoc cluster head. Both $\mathcal{S}$ and $\mathcal{D}$ are 135 granted access to the licensed spectrum, whereas the $N$ RNs 136 are not licensees. To simplify our investigations, we made the 137 following assumptions. All the channels involved are assumed 138 to undergo quasi-static Rayleigh fading; hence, the complex139 valued fading envelope remains constant during a transmission

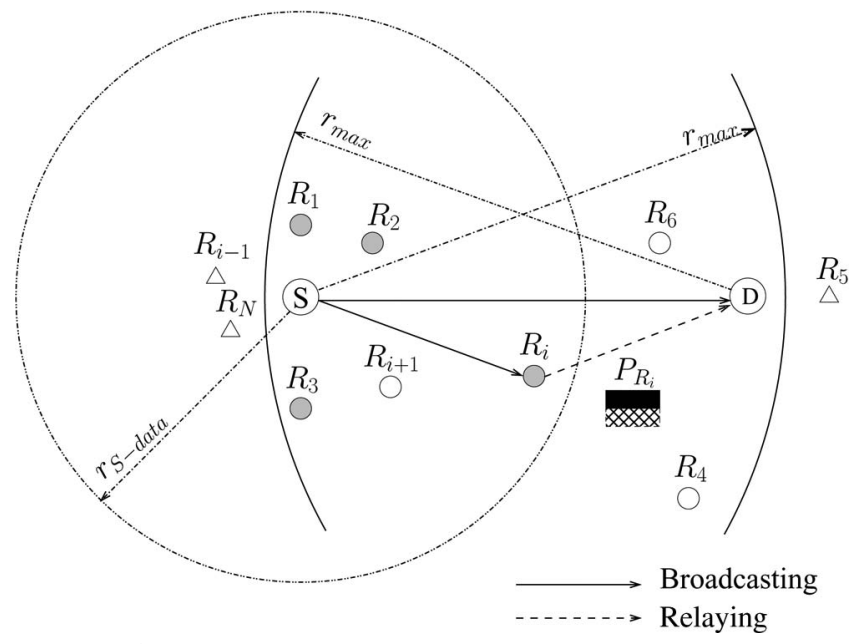

Fig. 1. Cooperative topology consists of one $\mathrm{SN} \mathcal{S}$, one $\mathrm{DN} \mathcal{D}$, and a total of $N$ RNs $\mathcal{R}=\left\{\mathcal{R}_{1}, \ldots, \mathcal{R}_{N}\right\}$.

burst, ${ }^{1}$ whereas it is faded independently between the consec- 140 utive transmission bursts. Within a given transmission burst, 141 the duplex bidirectional channels between a pair of actively 142 communicating nodes are assumed to be identical, whereas 143 the channels of any of the remaining links are independent. 144 We assume perfect channel estimation for all nodes concerning 145 their own channels, ${ }^{2}$ but no knowledge of the remaining links 146 is assumed. Additionally, the nodes' own position information 147 is perfectly known at each node. We consider the effects of 148 free-space path loss that is modeled by $\rho=\lambda^{2} / 16 \pi^{2} d^{\eta}$, where 149 $\lambda$ represents the wavelength, $d$ is the transmitter-to-receiver 150 distance and $\eta$ denotes the path-loss exponent, which is 2 . All 151 nodes are assumed to be limited by the same maximum transmit 152 power $P_{\max }$.

\section{B. Distributed WW Cooperative Framework}

1) SN's Behavior: Rather than relying on monetary remu- 155 neration, $\mathcal{S}$ in our DWWCF intends to lease part of its spec- 156 trum to the RNs in exchange for cooperatively supporting 157 the source's transmission. Based on the RN's assistance, $\mathcal{S} 158$ is capable of successfully conveying its data at a reduced 159 transmit power of $P_{\mathcal{S} \text {-data }}$ and an increased transmit rate 160 of $\alpha C_{\mathcal{S}, \mathcal{D}}^{\max }(\alpha \geq 1)$, which is the SN's target transmit rate. 161 In greater detail, $\alpha$ is the ratio of the desired and afford- 162 able throughput termed as the SN's "factor of greediness," 163 whereas $C_{\mathcal{S}, \mathcal{D}}^{\max }$ is the maximum achievable rate of the source- 164 to-destination (SD) link, which can be formulated as $C_{\mathcal{S}, \mathcal{D}}^{\max }=165$ $\log _{2}\left(1+\left(\rho_{\mathcal{S}, \mathcal{D}}\left|h_{\mathcal{S}, \mathcal{D}}\right|^{2} P_{\max } / P_{N}\right)\right)$, where $P_{N}$ is the power of 166 the additive white Gaussian noise, whereas $\left|h_{\mathcal{S}, \mathcal{D}}\right|$ denotes the 167 magnitude of the flat Rayleigh channel between $\mathcal{S}$ and $\mathcal{D}$. Fur- 168 thermore, $\rho_{\mathcal{S}, \mathcal{D}}$ is the free-space path-loss gain between $\mathcal{S}$ and 169 $\mathcal{D}$. If $\mathcal{S}$ cannot acquire any cooperative transmission assistance, 170 it directly transmits its data to $\mathcal{D}$ at a higher transmit power 171

\footnotetext{
${ }^{1}$ We define a transmission burst as a single transmission attempt, excluding any subsequent retransmission attempts.

${ }^{2}$ The effect of realistic imperfect channel estimation is evaluated in Section IV-F
} 
$172 P_{\mathcal{S}}^{n c}$ and lower transmit rate $R_{\mathcal{S}}^{n c}$. Hence, $\mathcal{S}$ has two Objective 173 Functions (OF) in our DWWCF, which may be formulated as

$$
\begin{aligned}
& \mathrm{OF}_{\mathcal{S} 1}=\max \left\{\xi_{\mathcal{S}} \cdot R_{\mathcal{S}}^{r e q}+\left(1-\xi_{\mathcal{S}}\right) \cdot R_{\mathcal{S}}^{n c}\right\} \\
& \mathrm{OF}_{\mathcal{S} 2}=\min \left\{\xi_{\mathcal{S}} \cdot P_{\mathcal{S}-\text { data }}+\left(1-\xi_{\mathcal{S}}\right) \cdot P_{\mathcal{S}}^{n c}\right\}
\end{aligned}
$$

174 subject to $R_{\mathcal{S}}^{r e q}=\alpha C_{\mathcal{S}, \mathcal{D}}^{\max }>R_{\mathcal{S}}^{n c}$ and $\alpha \geq 1$, as well as $175 P_{\mathcal{S} \text {-data }}<P_{\mathcal{S}}^{n c}$, where $\xi_{\mathcal{S}}$ denotes the cooperative probability 176 of SN.

177 2) RN's Behavior: According to our DWWCF, the RN has 178 an incentive to forward data for $\mathcal{S}$ for the sake of accessing 179 the SN's spectrum to convey its own traffic. The selfish RN $\mathcal{R}_{i}$ 180 reserves a certain fraction of $\beta C_{\mathcal{R}_{i}, \mathcal{D}}^{\max }(0<\beta<1)$ of the Relay181 to-Destination (RD) channel's capacity for conveying its own 182 traffic, where $\beta$ is the RN's "factor of greediness" and $C_{\mathcal{R}_{i}, \mathcal{D}}^{\max }$ is 183 given by: $C_{\mathcal{R}_{i}, \mathcal{D}}^{\max }=\log _{2}\left(1+\left(\rho_{\mathcal{R}_{i}, \mathcal{D}}\left|h_{\mathcal{R}_{i}, \mathcal{D}}\right|^{2} P_{\max } / P_{N}\right)\right)$, while $184\left|h_{\mathcal{R}_{i}, \mathcal{D}}\right|$ denotes the magnitude of the flat Rayleigh channel 185 between $\mathcal{R}_{i}$ as well as $\mathcal{D}$, and $\rho_{\mathcal{R}_{i}, \mathcal{D}}$ is the free-space path186 loss gain between $\mathcal{R}_{i}$ and $\mathcal{D}$. Based on our DWWCF, each $187 \mathrm{RN} \mathcal{R}_{i}$ carries out autonomous decisions concerning its own 188 cooperative strategy by optimizing its own OF, which may be 189 formulated as

$$
\mathrm{OF}_{\mathrm{RN} 1}=\max \left\{\xi_{\mathcal{R}_{i}} \cdot \beta C_{\mathcal{R}_{i}, \mathcal{D}}^{\max }\right\}
$$

190 subject to $0<\beta<1$, where $\xi_{\mathcal{R}_{i}}$ denotes the probability that $191 \mathrm{RN} \mathcal{R}_{i}$ is granted the transmission opportunity.

192 When the RNs provide cooperative transmission assis193 tance, extra energy is dissipated when relaying data for $\mathcal{S}$. 194 Hence, another OF is designed in our DWWCF to select the 195 best RN, which may be formulated as

$$
\mathrm{OF}_{\mathrm{RN} 2}=\min \sum_{i=1}^{N}\left\{\xi_{\mathcal{R}_{i}} \cdot P_{\mathcal{R}_{i}}\right\}
$$

196 subject to $\sum_{i=1}^{N} \xi_{\mathcal{R}_{i}} \leq 1$, and $P_{\mathcal{R}_{i}} \leq P_{\max }$, where $P_{\mathcal{R}_{i}}$ is the 197 RN's transmit power required for successfully forwarding the 198 SN's data and for simultaneously conveying its own data. Based 199 on the above OFs, it is quite a challenge to mathematically 200 solve these optimization problems in our DWWCF. Hence, we 201 designed a WW cooperative MAC protocol to implement our 202 DWWCF.

\section{III. Win-Win Cooperative Medium Access Control 204 PROTOCOL DESCRIPTION}

205 Based on the request-to-send/clear-to-send (RTS/CTS) sig206 naling of the legacy IEEE 802.11 protocol, a WW cooperative 207 MAC protocol is developed to implement our DWWCF, which 208 is formulated in Section II-B. The proposed signaling procedure 209 is detailed in Fig. 2, which includes three phases, as detailed in 210 the following.

\section{A. Phase I: Initialization}

212 Before $\mathcal{S}$ transmits any data frame, it issues an RTS message 213 to $\mathcal{D}$ at the maximum transmission power $P_{\max }$ to reserve the 214 shared channel, as shown in Fig. 2 . When $\mathcal{D}$ correctly receives 215 the RTS message, it replies with a CTS message, employing the

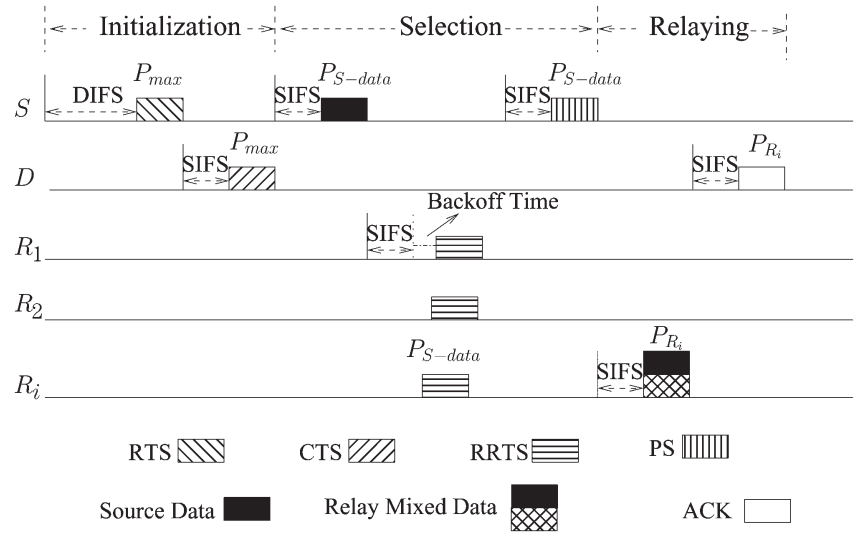

Fig. 2. Overall signaling procedure. RTS: Request-to-send. CTS: Clear-tosend. RRTS: Relay-request-to-send. PS: Please-send. ACK: Acknowledgement. DIFS: Distributed interframe space. SIFS: Short interframe space.

same transmission power $P_{\max }$. The instantaneous transmission 216 ranges of the sources are shown in Fig. 1. To elaborate a little 217 further, we include the transmitter's position information into 218 the RTS and CTS signaling frame; thus, any RNs in the set $\mathcal{R}, 219$ which can overhear both the RTS and CTS messages, will be 220 aware of the imminently forthcoming transmission and of the 221 position information on $\mathcal{S}$ and $\mathcal{D}$. Based on the knowledge of 222 their own position and on the position of the $\mathrm{SN}$ and the DN, 223 these RNs are capable of calculating the distances from both the 224 $\mathrm{SN}$ and the DN to themselves. These RNs, which are denoted 225 by filled or hollow circles in Fig. 1, form a potential cooperative 226 $\mathrm{RN}$ set $\mathcal{R}_{c} \subset \mathcal{R}$.

\section{B. Phase II: Relay Selection}

Following the initialization phase, the RN selection proce- 229 dure is constituted by a data transmission and two beacon 230 message exchanges, as detailed in the following.

231

1) Step I-Invitation for Cooperation: If $\mathcal{S}$ does not receive 232 a CTS message from $\mathcal{D}$, it would retransmit the RTS message as 233 specified in the legacy IEEE 802.11 protocol [22]. In contrast, if 234 $\mathcal{S}$ receives a CTS message from $\mathcal{D}$, it broadcasts its data frame 235 after a short interframe space (SIFS) interval at reduced power 236 of $P_{\mathcal{S} \text {-data }}$ and its target transmit rate of $\alpha C_{\mathcal{S}, \mathcal{D}}^{\max }(\alpha \geq 1)$, as 237 shown in Fig. 2. As a result, both $\mathcal{D}$ and the RNs in the set 238 $\mathcal{R}_{c}$ will hear this broadcast. When $\alpha$ is higher than unity, the 239 SN's data cannot be successfully transmitted to $\mathcal{D}$ in its entirety. 240 However, $\mathcal{D}$ will store this data frame and exploits the classic 241 Chase combining scheme [23] to combine it with the duplicated 242 data frame independently transmitted by the potential candidate 243 relays, for the sake of achieving rate improvements. Therefore, 244 the SN's aggregated rate achieved by using Chase combining 245 may be expressed as [24]

$$
\alpha C_{\mathcal{S}, \mathcal{D}}^{\max }=\log _{2}\left(1+\gamma_{\mathcal{S}, \mathcal{D}}^{(1)}+\gamma_{\mathcal{R}_{i}}^{\mathcal{S}}\right)
$$

subject to $\alpha \geq 1$, where $\gamma_{\mathcal{S}, \mathcal{D}}^{(1)}$ denotes the receiver's signal- 247 to-interference-plus-noise ratio (SINR) related to the direct 248 transmission during the broadcast phase. Furthermore, $\gamma_{\mathcal{R}_{i}}^{\mathcal{S}} 249$ represents the receive SINR of the SN's data frame, which is 250 transmitted during the relaying phase to be introduced. Based 251 


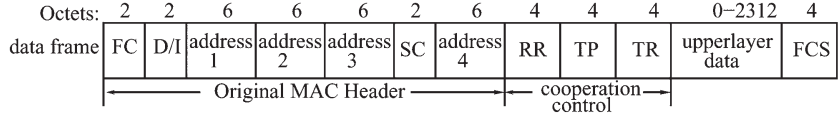

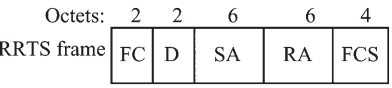

$$
\begin{aligned}
& \begin{array}{rl|l|l|l|}
\text { Octets: } & 2 & 2 & 6 & 4 \\
\text { PS frame } & \text { FC } & \text { D } & \text { BRA } & \text { FCS } \\
\hline
\end{array}
\end{aligned}
$$

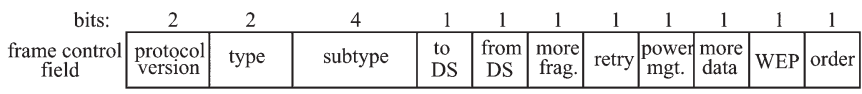

$$
\begin{aligned}
& \mathrm{FC}=\text { frame control } \quad \mathrm{D} / \mathrm{I}=\text { duration } / \mathrm{ID} \quad \mathrm{SC}=\text { sequence control } \quad \mathrm{RR}=\text { relay requirement } \\
& \mathrm{TP}=\text { transmit power } \quad \mathrm{TR}=\text { transmit rate } \mathrm{FCS}=\text { frame check sequence } \mathrm{D}=\text { duration } \\
& \mathrm{DS}=\text { distribution system } \mathrm{RA}=\text { relay address } \quad \mathrm{SA}=\text { source address } \quad \mathrm{BRA}=\text { best relay address }
\end{aligned}
$$

Fig. 3. Formats of the data frames, the RRTS message, and the PS message.

252 on the estimated channel state information (CSI) of the SD 253 link, $\mathcal{S}$ first calculates the receive SINR of $\gamma_{\mathcal{S}, \mathcal{D}}^{(1)}$ achieved by 254 the direct transmission during the broadcast phase. Then, based 255 on $\gamma_{\mathcal{S}, \mathcal{D}}^{(1)}$ and (5), $\mathcal{S}$ calculates the receive SINR of $\gamma_{\mathcal{R}}^{\mathcal{S}}$, which 256 must be guaranteed by the best $\mathrm{RN}$ and includes the value of $257 \gamma_{\mathcal{R}_{i}}^{\mathcal{S}}$ into the relay requirement (RR) field of its data frame for 258 implicitly informing the RNs of the SN's transmit requirement $259 \alpha C_{\mathcal{S}, \mathcal{D}}^{\max }$. The RNs in the vicinity, which correctly receive the 260 SN's data frame, are capable of inferring the value of $\gamma_{\mathcal{R}_{i}}^{\mathcal{S}}$ by 261 reading the RR field of the appropriately designed cooperative 262 MAC data frame, as shown in Fig. $3 .^{3}$

263 2) Step II-Contend for Cooperation: For clarity, we break 264 the discussion of this step into several subtopics, namely, the 265 cooperative decision, the backoff algorithm, and contention 266 message derivation.

267 Cooperation decision: If a particular $\mathrm{RN} \mathcal{R}_{i} \in \mathcal{R}_{c}$ erroneously 268 receives the data frame from $\mathcal{S}, \mathcal{R}_{i}$ would drop this data 269 frame and would keep on sensing the channel, as shown in Table I. On the other hand, if cooperative $\mathrm{RN} \mathcal{R}_{i} \in$ $\mathcal{R}_{c}$ correctly receives a data frame from $\mathcal{S}$, it calculates the transmit power $P_{\mathcal{R}_{i}}^{\mathcal{S}}$ necessitated to satisfy the $\mathrm{SN}$ rate requirement and the transmit power $P_{\mathcal{R}_{i}}^{\mathcal{R}}$ required to guarantee a throughput of $\beta C_{\mathcal{R}_{i}, \mathcal{D}}^{\max }$. If the sum of transmit power $P_{\mathcal{R}_{i}}=P_{\mathcal{R}_{i}}^{\mathcal{S}}+P_{\mathcal{R}_{i}}^{\mathcal{R}}$ is higher than $P_{\text {max }}, \mathcal{R}_{i}$ has to give up contending for the cooperative opportunity and drop this SN's data frame, as shown in Table I. On the other hand, if $P_{\mathcal{R}_{i}}$ does not exceed $P_{\max }, \mathcal{R}_{i}$ would send a relay-request-to-send (RRTS) message to $\mathcal{S}$ after waiting for a SIFS interval and its backoff time, which is calculated based on the proposed backoff algorithm for the sake of contending for a transmission opportunity, as shown in Table I. The RRTS message in Fig. 2 informs $\mathcal{S}$ about the RN's correct reception and its intention to cooperate. Hence, the specific RNs, which decide to contend for the transmission opportunity form a smaller contending set of $\mathcal{R}_{c c} \in \mathcal{R}_{c}$. These RNs are represented by the filled circles in Fig. 1. It is noted that the value of $P_{\mathcal{R}_{i}}$ is not included in the RRTS message in Fig. 3 since the proposed backoff

\footnotetext{
${ }^{3}$ Apart from the cooperative control fields of the data frame, as shown in Fig. 3, the remaining fields are the same as those of the data frame specified in the IEEE 802.11 standards [22].
}

TABLE I

Procedure of THE RN SUbMission CoOperative DeCISION

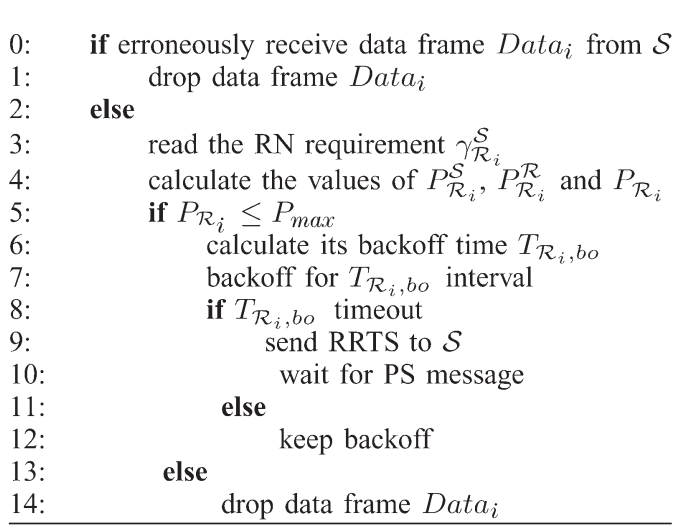

algorithm can identify the different values of $P_{\mathcal{R}_{i}}$ promised 290 by the contending RNs.

291

Backoff algorithm: To minimize the total transmit power of 292 the RNs, which is formulated by (4), we design a backoff 293 algorithm to select the best RN. As shown in Fig. 2, before 294 issuing the RRTS message, the RN $\mathcal{R}_{i} \in \mathcal{R}_{c c}$ has to wait 295 for a SIFS interval and for subsequent backoff duration 296 of $T_{\mathcal{R}_{i}}$,bo, which is defined as $T_{\mathcal{R}_{i}, \text { bo }}=\varphi_{\mathcal{R}_{i}} T_{w}$, where 297 $T_{w}=\mathrm{CWmin} \cdot$ SlotTime is the contention window $(\mathrm{CW}) 298$ length, ${ }^{4}$ with $\mathrm{CWmin}$ being the minimum $\mathrm{CW}$ duration 299 specified in the IEEE802.11 standards [22]. The coefficient 300 $\varphi_{\mathcal{R}_{i}}$ is defined as $\varphi_{\mathcal{R}_{i}}=P_{\mathcal{R}_{i}^{\min }} / P_{\max }$. Hence, the specific 301 candidate RN, which promises the lowest transmit power, 302 may first transmit its RRTS message as a benefit of its 303 shortest backoff time. In each RN selection phase, $\mathcal{S}$ has 304 to wait for a fixed period of ( $T_{w}+$ SlotTime $)$ to collect the 305 responses of the potential candidate RNs. If $\mathcal{S}$ correctly re- 306 ceives the RRTS message before its fixed waiting duration 307 times out, it selects the transmitter of that specific RRTS, 308 which was the first one to be correctly received as the 309 best RN, without considering the RRTS messages arriving 310 later and without comparing the specific transmit power 311 promised by the individual candidate RNs. Hence, the best 312 $\mathrm{RN}$ is selected in a distributed manner both without a cen- 313 tralized controller and without any information exchange 314 between the candidate RNs. Since the value of $P_{\mathcal{R}_{i}^{\min }} 315$ promised by the candidate $\mathrm{RN} \mathcal{R}_{i}$ is always lower than 316 $P_{\max }$, the backoff time allocated to $\mathcal{R}_{i}$ will not exceed the 317 SN's fixed waiting duration of ( $T_{w}+$ SlotTime $)$. Hence, all 318 the candidate RNs may issue their RRTS messages before 319 $\mathcal{S}$ stops waiting for the responses.

Contention message derivation: According to our backoff al- 321 gorithm, the specific RN promising the lowest power may 322 be granted the transmission opportunity to minimize the 323 total transmit power of RNs. Hence, the greedy RN has 324 to minimize its transmit power by only satisfying its rate 325 requirement of $\beta C_{\mathcal{R}_{i}, \mathcal{D}}^{\max }$ to wait for a shorter backoff time, 326

\footnotetext{
${ }^{4}$ In the IEEE 802.11 standard, a SlotTime consists of the time required to physically sense the medium and to declare the channel as "clear," as well as the MAC processing delay, the propagation delay, and the "receiver/transmitter turn-around time," which is the time required for the physical layer to change from receiving to transmitting at the start of the first bit [22].
} 
which is calculated based on the proposed backoff algorithm. Therefore, we have

$$
P_{\mathcal{R}_{i}^{\min }}\left(P_{\mathcal{R}_{i}}^{\mathcal{S}}, P_{\mathcal{R}_{i}^{\min }}^{\mathcal{R}} \mid \alpha, \beta\right)=P_{\mathcal{R}_{i}}^{\mathcal{S}}+P_{\mathcal{R}_{i}^{\min }}^{\mathcal{R}}
$$

subject to the condition of $C_{\mathcal{R}_{i}}^{\mathcal{R}}=\beta C_{\mathcal{R}_{i}, \mathcal{D}}^{\max }$ and $\alpha>1$, as well as $0<\beta<1$.

331 Let us now consider how to find $P_{\mathcal{R}_{i}}^{\mathcal{S}}$ and $P_{\mathcal{R}_{i}^{\min }}^{\mathcal{R}}$ of (6). In 332 our design, the RN employs SPC for jointly encoding both the 333 SN's and its own data. $\mathcal{D}$ then extracts the SN's data from 334 the relayed composite signal with the aid of SIC. Finally, the 335 extracted relayed component and the direct component are 336 combined. Assuming that $\mathcal{D}$ treats the RN's data frame as 337 interference, the receive SINR $\gamma_{\mathcal{R}_{i}}^{\mathcal{S}}$ of the SN's data frame re338 layed by the $\mathrm{RN}$ is given by $\gamma_{\mathcal{R}_{i}}^{\mathcal{S}}=\left(\rho_{\mathcal{R}_{i}, \mathcal{D}}\left|h_{\mathcal{R}_{i}, \mathcal{D}}\right|^{2} P_{\mathcal{R}_{i}}^{\mathcal{S}}\right) /\left(P_{N}+\right.$ $\left.339 \rho_{\mathcal{R}_{i}, \mathcal{D}}\left|h_{\mathcal{R}_{i}, \mathcal{D}}\right|^{2} P_{\mathcal{R}_{i}}^{\mathcal{R}}\right)$. After successfully retrieving the SN's data 340 frame, $\mathcal{D}$ becomes capable of decoding the RN's data frame by 341 removing the SN's interference with the aid of a SIC scheme 342 [25]. Hence, the achievable rate of the RN may be formulated as $343 C_{\mathcal{R}_{i}}^{\mathcal{R}}=\log _{2}\left(1+\left(\rho_{\mathcal{R}_{i}, \mathcal{D}}\left|h_{\mathcal{R}_{i}, \mathcal{D}}\right|^{2} P_{\mathcal{R}_{i}}^{\mathcal{R}} / P_{N}\right)\right)$. According to the 344 relaying strategy employed, the RN calculates the minimum 345 power required for the rate $C_{\mathcal{R}_{i}}^{\mathcal{R}}$ to reach $\beta C_{\mathcal{R}_{i}, \mathcal{D}}^{\max }$. Thus, the 346 value of $P_{\mathcal{R}_{i}^{\min }}^{\mathcal{R}}$ is explicitly given as $P_{\mathcal{R}_{i}^{\min }}^{\mathcal{R}}=\left(\left(2^{\beta C_{\mathcal{R}_{i}, \mathcal{D}}^{\max }}\right.\right.$ $\left.3471) P_{N}\right) /\left(\rho_{\mathcal{R}_{i}, \mathcal{D}}\left|h_{\mathcal{R}_{i}, \mathcal{D}}\right|^{2}\right)$, which is subjected to $0<\beta<1$. 348 Likewise, based on the metrics of $\gamma_{\mathcal{R}_{i}}^{\mathcal{S}}$ and $P_{\mathcal{R}_{i}^{\min }}^{\mathcal{R}}$, the RN 349 is capable of calculating the transmit power $P_{\mathcal{R}_{i}}{ }^{i}$ required for 350 successfully delivering the SN's data at a throughput of $\alpha C_{\mathcal{S}, \mathcal{D}}^{\max }$, 351 which is given by $P_{\mathcal{R}_{i}}^{\mathcal{S}}=\gamma_{\mathcal{R}_{i}}^{\mathcal{S}}\left(\left(P_{N} / \rho_{\mathcal{R}_{i}, \mathcal{D}}\left|h_{\mathcal{R}_{i}, \mathcal{D}}\right|^{2}\right)+P_{\mathcal{R}_{i}^{\min }}^{\mathcal{R}}\right)$, 352 where $\gamma_{\mathcal{R}_{i}}^{\mathcal{S}}$ has been given in Step I. Based on the given 353 derivation, $\mathcal{R}_{i}$ calculates the value of $P_{\mathcal{R}_{i}}^{\min }$ as the sum of $P_{\mathcal{R}_{i}}^{\mathcal{S}}$ 354 and $P_{\mathcal{R}_{i}^{\min }}^{\mathcal{R}}$.

355 3) Step III-Accept for Cooperation: After waiting for the 356 fixed duration of $\left(T_{w}+\right.$ SlotTime $)$ specified by the proposed 357 backoff algorithm and for a subsequent SIFS interval, $\mathcal{S}$ replies 358 to the best RN $\mathcal{R}_{\hat{i}}$ associated with the first RRTS message that 359 was correctly received by sending a please-send (PS) message if $360 \mathcal{S}$ correctly received the RRTS message during its fixed waiting 361 period of $\left(T_{w}+\right.$ SlotTime $)$, as shown in Fig. 2 and Table II. The 362 format of the PS frame is characterized in Fig. 3. Since the SN 363 sends its data frame and PS message at the same transmission 364 power of $P_{\mathcal{S} \text {-data }}$, all the RNs, which have correctly received 365 the data frame from the SN will overhear the PS message. This 366 guarantees that only the best $\mathrm{RN}$ forwards its data frame to $\mathcal{D}$ 367 during the data-forwarding phase.

\section{C. Phase III: Cooperative Transmission}

369 In this phase, the best RN $\mathcal{R}_{\hat{i}}$ forwards the superimposed SR 370 data to $\mathcal{D}$ if $\mathcal{S}$ successfully selects the best RN. Otherwise, $\mathcal{S}$ 371 retransmits its data frame to $\mathcal{D}$, as shown in Fig. 2 and Table II. 372 1) Data Forwarding and Relay Retransmission: If RN $\mathcal{R}_{i} \in$ $373 \mathcal{R}_{c c}$ finds that the receiver of the received PS message is not 374 itself, it would drop the SN's data and would keep on sensing 375 the medium. On the other hand, if the $\mathrm{RN} \mathcal{R}_{i} \in \mathcal{R}_{c c}$ received 376 a PS message that is destined for itself, it will encode both the 377 SN's and its data with the aid of SPC and will forward the super-
TABLE II

PROCEDURE OF SN

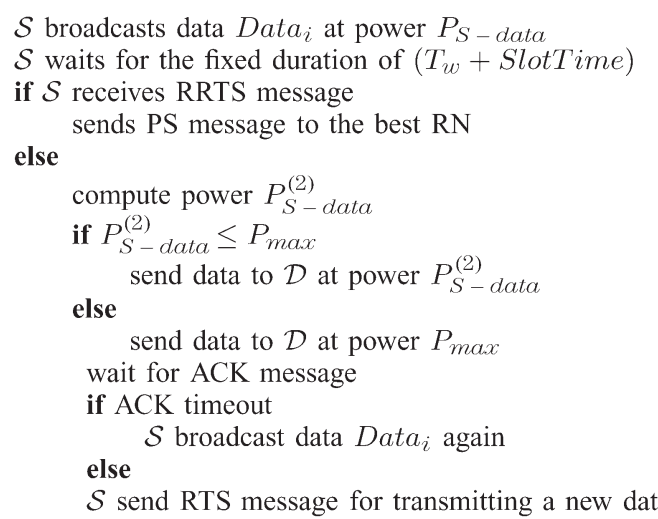

imposed SR data frame to $\mathcal{D}$ at its precalculated transmission 378 power of $P_{\mathcal{R}^{\min }}$ after an SIFS period, acting as the best RN, 379 as shown in Fig. 2. Finally, at the DN, the classic automatic 380 repeat request procedure will be initiated, when receiving the 381 forwarded data and successfully decoding and combing it with 382 the most recent direct transmission during Step I of Phase II. 383

2) Source Retransmission: If none of the RNs competes for 384 a transmission opportunity or multiple RRTS messages collided 385 at the $\mathrm{SN}, \mathcal{S}$ directly sends its data to $\mathcal{D}$ as a replica without 386 relaying. This transmission takes place either at the specific 387 transmit power of $P_{\mathcal{S} \text {-data }}^{(2)}$, which is capable of guaranteeing 388 the expected rate of $\alpha C_{\mathcal{S}, \mathcal{D}}^{\max }$, or failing that, it resorts to using 389 the maximum affordable transmit power of $P_{\max }$, as shown 390 in Table II. If $\mathcal{D}$ receives this data frame, it replies with an 391 acknowledgment (ACK) message to $\mathcal{S}$ after successfully de- 392 coding and combining the frame with the most recent erroneous 393 data frame broadcast by $\mathcal{S}$. If $\mathcal{S}$ does not receive any response 394 from $\mathcal{D}$ before the timer set for waiting for an ACK message 395 is expired, it will broadcast its data again at power of $P_{\mathcal{S}-\text { data }} 396$ to seek cooperation, and the RN selection procedure described 397 earlier is repeated, as shown in Table II.

\section{Simulation Results}

To evaluate the achievable performance of the proposed 400 scheme, we present our simulation results based on Omnet++. 401 Based on the network model introduced in Section II-A, we 402 consider two scenarios to investigate both the achievable rate 403 and EC improvement, and to analyze the RN's behavior. 404 In the first scenario, all the RNs are randomly distributed across 405 the entire network area, whereas $\mathcal{S}$ and $\mathcal{D}$ have fixed positions. 406 The network size considered ranges from $u=5$ nodes to $u=407$ 30 nodes for the sake of evaluating the influence of the size 408 of the networks on the achievable rate and EC. In the other sce- 409 nario, we consider a small network supporting $u=5$ nodes, i.e., 410 $\mathcal{S}, \mathcal{D}$, and three RNs, where all the nodes have fixed positions. 411 One of the three RNs is located at the position of $d=1 / 4$ along 412 the SD link. Another RN is in the middle of the SD link at 413 $d=1 / 2$, whereas the third $\mathrm{RN}$ is at the point $d=3 / 4$ of the SD 414 link. In the given two scenarios, the values of $P_{\max }$ and $P_{\mathcal{S} \text {-data }} 415$ are 2 and $1 \mathrm{~mW}$, respectively. The size of CWmin is 7, whereas 416 SlotTime is set to $20 \mu \mathrm{s}$. Furthermore, the length of SIFS is 417 


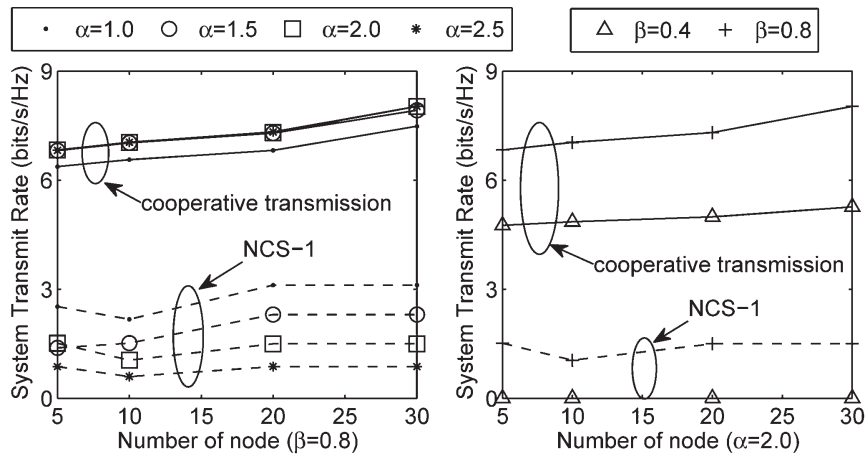

Fig. 4. System's total achievable rate improvement.

$41810 \mu \mathrm{s}$. The length of the data frame generated at the application 419 layer is $1024 \mathrm{~B}$. The length of the RRTS and PS messages is $42020 \mathrm{~B}$ and $14 \mathrm{~B}$, respectively, whereas that of the RTS and $421 \mathrm{CTS}$ is 24 and $18 \mathrm{~B}$. The greedy factor $\alpha$ ranges from 1 to 3 , 422 whereas the value of $\beta$ ranges from 0 to 0.8 . Both $\alpha$ and $\beta$ are 423 predetermined for each simulation.

424 Two noncooperative systems are introduced as the bench425 markers of our comparisons. We compare the system's achiev426 able total transmit rate (TTR) constituted by the sum of the 427 SN's and RN's transmit rate to that of the noncooperative 428 system 1 (NCS-1), which consumes the same total transmission 429 energy as our CSLS (WW-CSLS). Additionally, we compare 430 the total transmission EC to that of the noncooperative system 2 431 (NCS-2), which is capable of achieving the same TTR as our 432 WW-CSLS. Since the SN's data is transmitted twice by itself 433 and additionally by the best RN, if the cooperative transmission 434 is successful, two direct transmission phases are exploited in 435 both NCS- 1 and NCS-2. When aiming for investigating the 436 effect of our relay selection scheme, we compare the achievable 437 performance of our WW-CSLS to that of a random CSLS 438 (Ran-CSLS), where the best RN is randomly selected with439 out considering the transmit power required for providing a 440 successful cooperative transmission. To evaluate their perfor441 mance, we adopt the idealized simplifying assumption that the 442 control messages are received without errors in both NCS-1 443 and NCS-2, as well as in WW-CSLS. In Sections IV-E and F, 444 we investigated a more practical network.

\section{A. Effect of Cooperative Transmission}

446 Let us now investigate the effects of cooperative transmission 447 on the TTR and EC by comparing the performance achieved in 448 the first scenario and NCS-1 and in NCS-2.

449 1) Achievable Transmit Rate: Fig. 4 compares the system's 450 TTR, namely, the sum of both the SN's rate and the RN's rate 451 achieved by the WW-CSLS relying on our WW cooperative 452 MAC protocol to that of NCS-1. It is observed in Fig. 4 that, 453 as expected, the system's achievable TTR relying on our WW454 CSLS is higher than $6 \mathrm{bit} / \mathrm{s} / \mathrm{Hz}$, even for $\alpha=1$ and $\beta=0.8$, 455 which is more than twice as high as that achieved by NCS- 1 , 456 which consumes the same total transmission energy, given the 457 same values of $\alpha$ and $\beta$. Additionally, for $\beta=0.4$ and $\alpha=2$, 458 the system's TTR achieved by our WW-CSLS is in excess of $4594 \mathrm{bit} / \mathrm{s} / \mathrm{Hz}$, while in fact, no successful transmissions may be

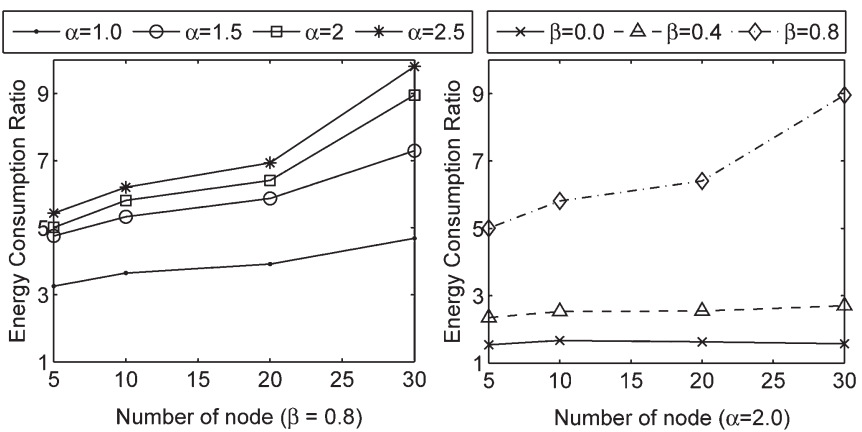

Fig. 5. Energy consumption ratio of $E_{\text {noncoop }} / E_{\text {coop }}$.

supported in NCS-1 for the same values of $\alpha$ and $\beta$ due to 460 the system's low EC. Hence, the proposed WW cooperative 461 MAC protocol is capable of providing a considerable TTR im- 462 provement, despite consuming low energy. As shown in Fig. 4, 463 the system's TTR achieved by our WW-CSLS is increased, 464 when $\mathcal{S}$ becomes greedier due to the SN's increased transmit 465 rate requirement. Additionally, when $\beta$ is increased, the best 466 RN will be rewarded by a considerably higher rate for its own 467 traffic, provided that the cooperation is successful. Hence, the 468 system's TTR is increased, when the RN becomes greedier, 469 as shown in Fig. 4. Moreover, the achievable TTR of our 470 WW-CSLS is gradually increased, when the network becomes 471 larger. The above investigations imply that the proposed WW 472 cooperative MAC protocol is capable of providing significant 473 TTR improvements.

474

2) Energy Consumption: Fig. 5 shows the achievable EC 475 ratio (ECR) of $E_{\text {noncoop }} / E_{\text {coop }}$, where $E_{\text {coop }}$ denotes the sys- 476 tem's total transmission $\mathrm{EC}^{5}$ for our cooperative MAC protocol 477 and $E_{\text {noncoop }}$ represents that of NCS-2, which is capable of 478 achieving the same system's TTR as our WW-CSLS. As shown 479 in Fig. 5, compared with NCS-2, two third of the system's 480 total energy may be saved by exploiting the proposed WW 481 cooperative MAC protocol, given $\beta=0.8$. The EC $E_{\text {coop }}$ of 482 our WW-CSLS is reduced when $\mathcal{S}$ becomes greedier, which 483 can be also characterized by the TTR of NCS-1 in Fig. 4. 484 By contrast, the EC $E_{\text {noncoop }}$ of NCS-2 is slightly increased, 485 when $\mathcal{S}$ becomes greedier due to the slightly increased system 486 rate of WW-CSLS. Hence, the ECR is increased, when $\mathcal{S} 487$ becomes greedier, as shown in Fig. 5. As $\beta$ is increased, the 488 system's ECR is increased from 1.5 to 5 for $\alpha=2$ and $u=5,489$ as shown in Fig. 5. When the RNs become greedier, fewer 490 RNs can afford the increased power required for successfully 491 forwarding the SPC data. However, the transmit rate achieved 492 by the best RN is considerably increased. Hence, an increased 493 total energy is required by NCS-2 for the sake of achieving the 494 same system rate as our WW-CSLS. Therefore, the system's 495 ECR of $E_{\text {noncoop }} / E_{\text {coop }}$ is increased when the RN becomes 496 greedier. Based on the given discussions, the proposed WW co- 497 operative MAC protocol is capable of achieving a considerable 498 system rate improvement while offering a satisfactory energy 499 efficiency.

\footnotetext{
${ }^{5}$ It is reasonable to focus on the transmission EC and ignore the circuit processing EC in a large network where the transmission EC is dominant in the total EC [26].
} 

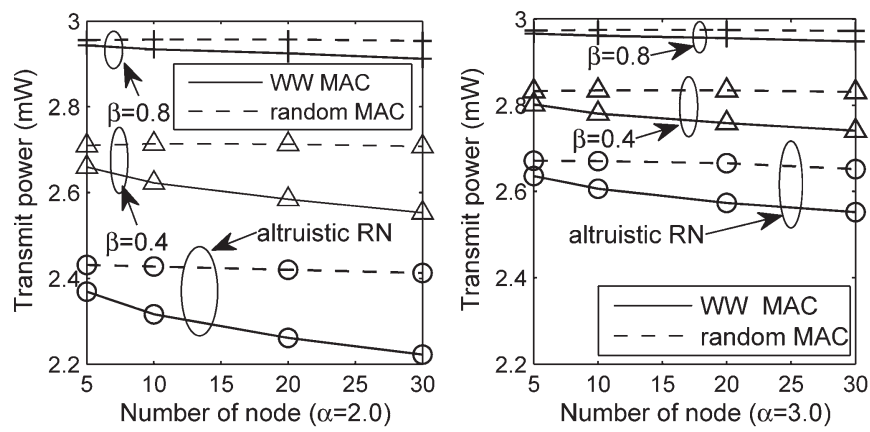

Fig. 6. System data transmit power consumed by our WW-CSLS and Ran-CSLS

\section{B. Effect of Relay Selection}

502 Let us now investigate the effect of the proposed RN selec503 tion scheme by evaluating the achievable performance of our 504 WW-CSLS and Ran-CSLS, where the best RN is randomly 505 selected.

506 1) Transmit Power: According to the proposed WW co507 operative MAC protocol, the specific RN that promises the 508 lowest transmit power $P_{\mathcal{R}_{i}}$ required for successfully conveying 509 superposition-coded data is selected as the best RN. However, 510 the best RN is randomly selected in Ran-CSLS without consid511 ering any system parameters, such as the transmit power $P_{\mathcal{R}_{i}}$. 512 Hence, the RN's transmit power $P_{\mathcal{R}_{i}}$ is the crucial parameter for 513 investigating the effect of the proposed RN selection scheme. 514 Fig. 6 quantifies the system's total data transmit power (TDTP) 515 for our WW-CSLS and that is consumed in Ran-CSLS. The 516 system's TDTP is defined as the sum of the SN's transmit power 517 required for conveying its data plus the RN's transmit power 518 necessitated for delivering the superposition-coded data.

519 Based on the proposed backoff algorithm, the system's TDTP 520 consumed in the WW-CSLS is lower than that of the Ran521 CSLS, as shown in Fig. 6. When the SN or RN becomes greed522 ier, less RNs can afford the increased transmit power required 523 to provide successful cooperative transmission assistance. This 524 phenomenon increases the probability that the same RN is 525 selected as the best RN in both WW-CSLS and Ran-CSLS. 526 Hence, the difference between the TDTP of our WW-CSLS and 527 that of Ran-CSLS is reduced when either $\alpha$ or $\beta$ is increased, 528 as shown in Fig. 6. Moreover, the TDTP of both WW-CSLS 529 and of the Ran-CSLS is reduced when the network hosts more 530 RNs due to the increased probability of having RNs, which 531 promise to reduce the transmit power in comparison with a 532 smaller network. However, the probability of the event that a 533 low-quality RN, namely, one which requires a higher transmit 534 power than other RNs, is selected as the best RN in the Ran535 CSLS is increased, when the network becomes larger. Hence, 536 compared with Ran-CSLS, an increased TDTP is saved by our 537 WW-CSLS when the network's size is increased.

538 2) Achievable Transmit Rate: Fig. 7 compares the system's 539 TTR, namely, the sum of both the SN's rate and the RN's rate 540 achieved by our WW-CSLS to that achieved by Ran-CSLS. 541 As shown in Fig. 7, the system's achievable TTR relying on $542 \mathrm{WW}-\mathrm{CSLS}$ is $8 \mathrm{bit} / \mathrm{s} / \mathrm{Hz}$ for $\beta=0.8$ and $u=30$, whereas a 543 lower TTR of $6.5 \mathrm{bit} / \mathrm{s} / \mathrm{Hz}$ is achieved by Ran-CSLS, given $\beta$ 544 and the network size. Compared with Ran-CSLS, the system's

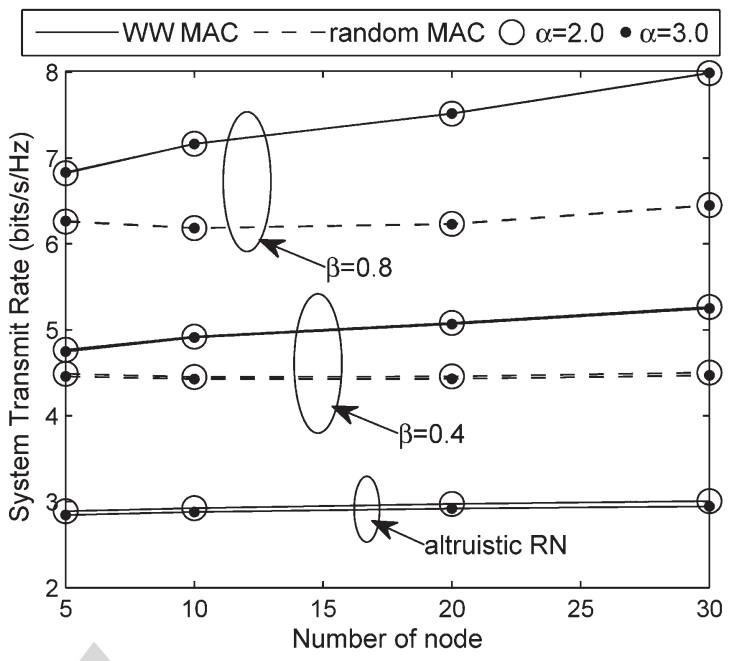

Fig. 7. System's total achievable rate improvement of our WW-CSLS and Ran-CSLS.

TTR can be improved by our WW-CSLS, even for lower $\beta 545$ values and for smaller networks, e.g., for $\beta=0.4$ and $u=5,546$ as shown in Fig. 7. Based on WW-CSLS, the specific RN that 547 promises lower transmit power of $P_{\mathcal{R}_{i}}$ may achieve a higher 548 transmit rate of $\beta C_{\mathcal{R}_{i}, \mathcal{D}}^{\max }$ due to having an improved RD link. 549 Hence, compared with Ran-CSLS, a higher TTR is achieved 550 by our WW-CSLS relying on selecting the specific RN, which 551 promises the lowest transmit power $P_{\mathcal{R}_{i}}$. 552

Observe in Fig. 7 that the proposed WW cooperative MAC 553 protocol is capable of providing a higher TTR improvement 554 than Ran-CSLS, when $\beta$ is increased. When an RN be- 555 comes greedier, its target transmit rate is increased. This phe- 556 nomenon increases the difference between the RN's transmit 557 rate achieved by WW-CSLS and that achieved by Ran-CSLS 558 when the RN that suffers from a low-quality RD link is selected 559 by Ran-CSLS. Hence, the difference between the TTR of WW- 560 CSLS and that of Ran-CSLS is increased when the RN becomes 561 greedier. Considering the CSLS, where the RN altruistically 562 forwards data for $\mathcal{S}$, the system's TTR is equal to the SN's rate. 563 Hence, the system's TTR remains the same, regardless of which 564 particular candidate RN is selected as the best RN when the 565 RNs are altruistic, as shown in Fig. 7.

566

As shown in Fig. 7, the system's TTR achieved by our WW- 567 CSLS is increased, when the network becomes larger. However, 568 the effect of the network's size on the TTR achieved by Ran- 569 CSLS is not as obvious as that on our WW-CSLS. When the 570 network hosts more RNs, the number of candidate RNs may 571 be increased. This phenomenon increases the probability that 572 a low-quality $\mathrm{RN}$ having a lower transmit rate is selected as 573 the best RN in Ran-CSLS. However, these low-quality RNs 574 cannot win the cooperative transmission opportunity in our 575 WW-CSLS if the specific RN promising a reduced transmit 576 power also contends for the transmission opportunity. Hence, 577 a higher TTR improvement is provided by the proposed WW 578 cooperative MAC protocol, as the network becomes larger, 579 as shown in Fig. 7. The given investigations imply that the 580 proposed WW cooperative MAC protocol is capable of saving 581 a substantial amount of transmit power while simultaneously 582 


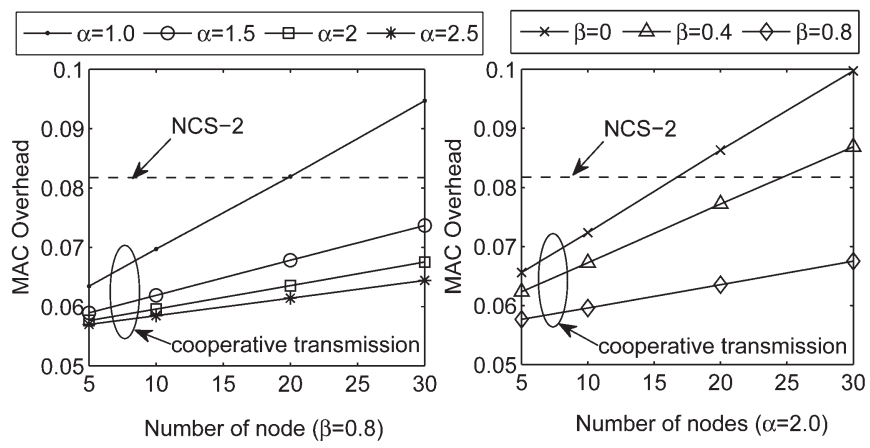

Fig. 8. MAC overhead for $\beta=0.8$ or $\alpha=2$.

583 providing significant TTR improvements compared with 584 Ran-CSLS.

\section{C. MAC Overhead}

586 Fig. 8 compares the MAC overhead of the proposed coop587 erative MAC protocol with that of NCS-2, which is based on 588 the RTS/CTS signaling regime of the IEEE 802.11 standards 589 [22]. The MAC overhead is defined as the ratio of $\left(\mathcal{N}_{\text {mac }-c}+\right.$ $\left.590 \mathcal{N}_{\text {mac }-h}+\mathcal{N}_{\text {mac }-t}\right) / \mathcal{N}_{\text {mac }-d}$, where $\mathcal{N}_{\text {mac }-c}$ denotes the num591 ber of bits of all MAC control messages, and $\mathcal{N}_{\text {mac }-h}$ and $592 \mathcal{N}_{\text {mac }-t}$ represent the number of header and tailing bits of the 593 MAC data frame, respectively. Furthermore, $\mathcal{N}_{\text {mac }-d}$ denotes 594 the number of bits in the payload data packet, including the 595 headers introduced by the higher layers. Observe in Fig. 8 that 596 the MAC overhead of the proposed WW cooperative MAC 597 protocol decreases, when either $\alpha$ or $\beta$ increases, because the 598 number of candidate RNs is reduced, whereas the $\mathrm{SN}$ or the $599 \mathrm{RN}$ becomes greedier. Compared with the traditional RTS/CTS 600 scheme specified in the IEEE 802.11 standards [22], the RRTS 601 message and the PS message are introduced into our WW-CSLS 602 to assist with RN selection if cooperation can be exploited. 603 However, compared with NCS-2, the RN's data can be also 604 transmitted with the aid of cooperation in WW-CSLS. Since 605 the length of the RN's data frames is higher than that of the 606 extra control messages, the MAC overhead introduced by our $607 \mathrm{WW}$ protocol is lower than that of the NCS-2 when the network 608 size is smaller than $u=20$. Although the overhead of our 609 WW-CSLS becomes higher than that of NCS-2 when the 610 network hosts more than $u=20$ nodes, the MAC overhead 611 introduced by our WW protocol always remains lower than 6120.1 for $\beta=0.8$ or $\alpha=2$.

\section{D. Relay Behavior}

614 To investigate the behavior of the relays, we analyze both the 615 transmission probability and the achievable rate improvement 616 of each RN for the configuration of $\alpha=2$ in the network 617 hosting $u=5$ nodes, as shown in Fig. 9(a) and (b). Upon 618 increasing $\beta$, the transmission probability of the RNs at " $d=$ $6191 / 4$ " and " $d=1 / 2$ " decreases, whereas that of the RN at 620 " $d=3 / 4$ " increases, as shown in Fig. 9(a). The $\mathrm{RN}$ at " $d=$ 621 3/4" always benefits from the highest transmission probability, 622 whereas the $\mathrm{RN}$ at " $d=1 / 4$ " has the lowest probability of 623 cooperative opportunities. As a benefit of its highest transmis624 sion probability, the $\mathrm{RN}$ at " $d=3 / 4$ " maintains the highest

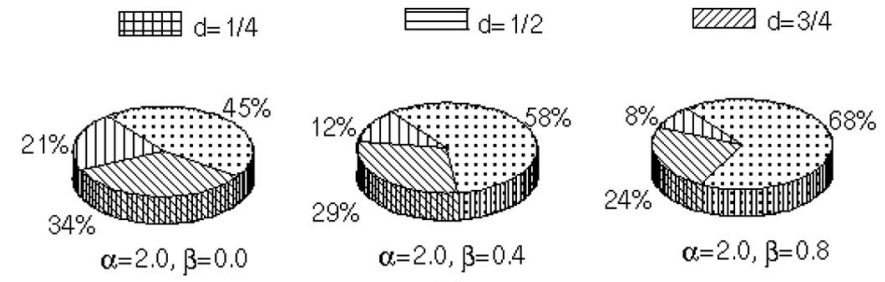

(a)

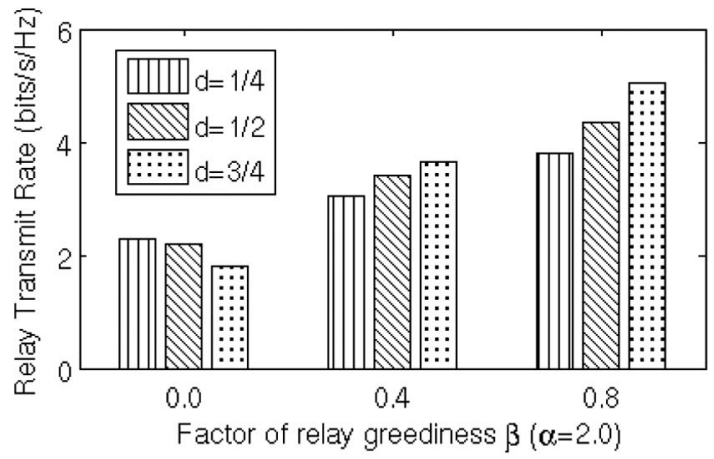

(b)

Fig. 9. RN transmission probability and the achievable rate improvement in a network hosting $u=5$ nodes, namely, $\mathcal{S}, \mathcal{D}$, and three RNs. (a) Relay transmission probability. (b) Relay achievable rate.

achievable rate improvement, which is above $5 \mathrm{bits} / \mathrm{s} / \mathrm{Hz}$ for 625 $\beta=0.8$ and $\alpha=2$. The achievable $\mathrm{RN}$-rate improvement at 626 " $d=1 / 4$ " is lower than that of the $\mathrm{RN}$ at " $d=1 / 2$," as shown in 627 Fig. 9(b). However, when the three RNs altruistically dedicate 628 themselves solely to forwarding data frames for $\mathcal{S}(\beta=0)$, the 629 achievable RN-rate improvement at " $d=1 / 4$ " is higher than 630 that of the other relays. Naturally, if the RNs become selfish, 631 their improved transmission probability leads to an increased 632 total throughput.

\section{E. Effect of Erroneous RTS Message}

The contention caused by hidden SNs or RNs may corrupt 635 the transmission of data and control messages. Apart from the 636 effects of corrupted RTS messages, the erroneous transmission 637 of both other control messages and of data have been considered 638 in our WW cooperative MAC protocol. Hence, the effect of 639 corrupted RTS messages on the system's transmit rate and on 640 the ECR of $E_{\text {rts-error }} / E_{\text {error-free }}$ that are achieved by our 641 WW-CSLS are evaluated, as shown in Fig. 10(a) and (b). The 642 variable $E_{\text {rts-error }}$ denotes the system's total EC for WW- 643 CSLS, where the RTS message may be corrupted. Furthermore, 644 $E_{\text {error-free }}$ is the system's total EC for WW-CSLS, where 645 error-free control messages are assumed. It is observed in 646 Fig. 10(a) and (b) that, when the RTS error probability is 647 increased, the system's TTR is decreased, and an increased 648 total system energy is dissipated by our WW-CSLS because 649 having more potentially erroneous RTS transmissions reduces 650 the probability of successful transmission, and the extra RTS 651 message retransmissions consume extra energy.

\section{F. Effect of Imperfect Channel Estimation}

To evaluate the overall system performance of our WW 654 cooperative protocol in a more practical scenario, we now 655 


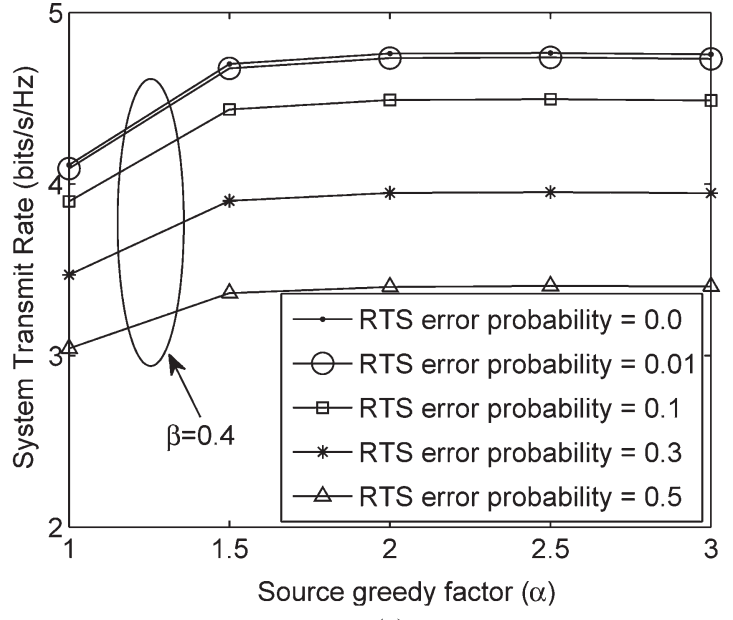

(a)

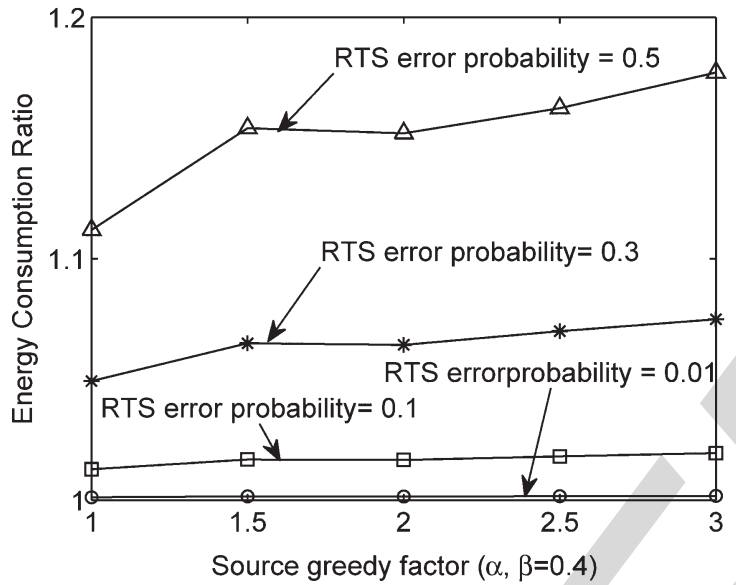

(b)

Fig. 10. System's total achievable transmit rate and system's ECR of $E_{\text {rts-error }} / E_{\text {error-free }}$ versus the SN's greedy factor parameterized with different RTS message error probabilities. (a) System's TTR. (b) System's ECR of $E_{\text {rts-error }} / E_{\text {error-free. }}$.

656 introduce Gaussion-distributed CSI estimation errors into our 657 WW-CSLS, instead of relying on the idealized simplifying 658 assumption of perfect CSI. The normalized mean square error 659 (NMSE) of the Gaussian channel estimation errors was defined 660 as $10 \log \left(E\left\{\|h-\hat{h}\|^{2}\right\} / E\left\{\|h\|^{2}\right\}\right)$ in decibels [27]. Compared 661 with the performance achieved by assuming perfect CSI, the 662 realistic imperfect channel estimation reduces the system's 663 attainable transmit rate and dramatically increases the system's 664 ECR of $E_{\text {error }} / E_{\text {perfect }}$, as shown in Fig. 11(a) and (b), respec665 tively. Variable $E_{\text {error }}$ denotes the system's energy consumed 666 by the CSLS relying on realistic imperfect channel estimation, 667 whereas $E_{\text {perfect }}$ denotes when perfect CSI is assumed. Based 668 on the given discussions, it is necessary to develop a more 669 robust cooperative MAC protocol to reduce the impact of 670 realistic imperfect channel estimation.

\section{G. Effect of Either Superposition Coding or Frame Combining}

672 To evaluate the achievable TTR improvement jointly attained 673 by SPC and SIC, we compare the system's TTR achieved by 674 our WW-CSLS with that of the cooperative system operating 675 without exploiting these techniques, as shown in Fig. 12. Since 676 there are two data frames jointly conveyed by the RN to

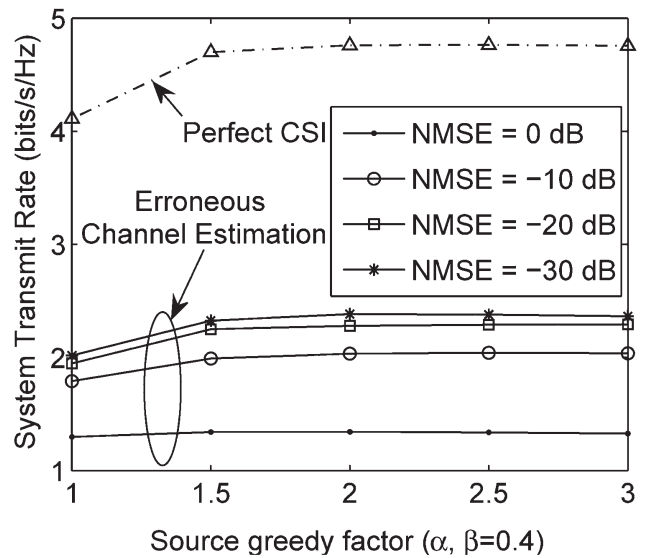

(a)

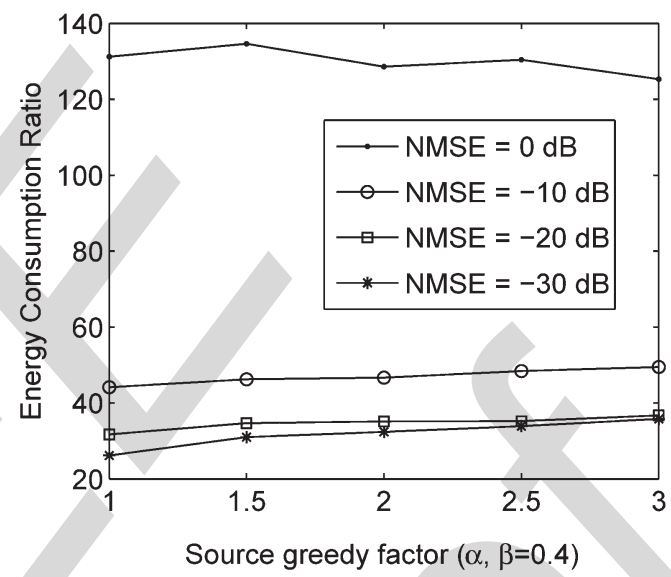

(b)

Fig. 11. System's total achievable transmit rate and system's ECR of $E_{\mathrm{rts}-\text { error }} / E_{\text {error-free }}$ versus the SN's greedy factor parameterized with different channel estimation NMSEs when $\beta=0.4$. (a) System's TTR. (b) System's ECR of $E_{\text {rts-error }} / E_{\text {error-free }}$.

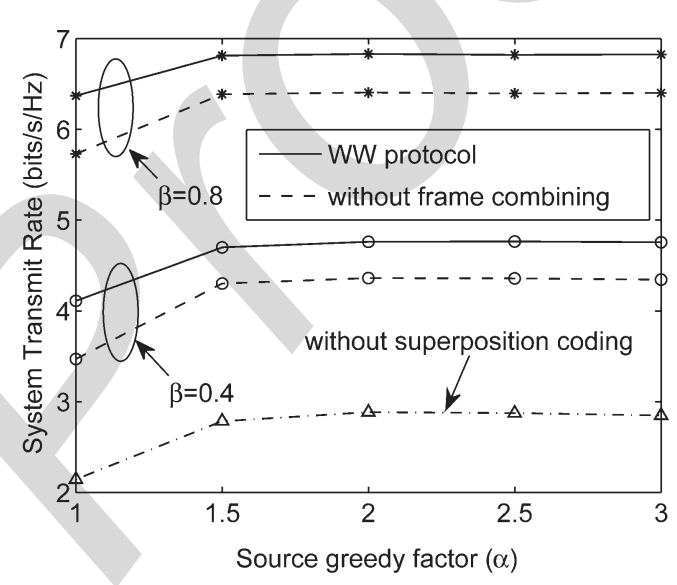

Fig. 12. System's total achievable transmit rate versus the SN's greedy factor both with and without SPC and SIC and frame combining.

$\mathcal{D}$ in our WW-CSLS, the best RN, which does not exploit 677 SPC, is assumed to forward only the SN's data instead of the 678 SPC data. As shown in Fig. 12, the system's TTR may be 679 increased from 2.9 to $6.9 \mathrm{bits} / \mathrm{s} / \mathrm{Hz}$ for $\alpha=2$ and $\beta=0.8$ by 680 jointly exploiting the SPC and SIC. Hence, these techniques are 681 capable of significantly improving the system's transmit rate. 682 To improve the SN's transmit rate, $\mathcal{D}$ invokes frame combining 683 
684 for amalgamating both the direct and relayed SN data after 685 successfully separating the SN's and RN's data. Fig. 12 shows 686 the system's TTR improvement achieved by exploiting frame 687 combining.

\section{CONClusion}

689 In this paper, we have formulated a distributed WW cooper690 ative framework for striking a tradeoff between the achievable 691 system rate improvement and EC and for granting transmission 692 opportunities for the unlicensed RNs. Furthermore, a WW 693 cooperative MAC layer protocol was proposed for implement694 ing our DWWCF. When compared with the corresponding 695 noncooperative system, the proposed scheme is capable of 696 providing a considerable transmit rate and transmission EC 697 improvements. This was achieved with the aid of joint SPC at 698 the RN for both the SN's and RN's data and by combining the 699 SD and RD signals at the DN. Our future work will consider 700 similar interference-limited scenarios relying on a more robust 701 cooperative MAC design.

702

703

704

705

706

707

708

709

710

711

712

713

714

715

716

717

718

719

720

721

722

723

724

725

726

727

728

729

730

731

732

733

734

735

736

\section{REFERENCES}

[1] L. Hanzo, Y. Akhtman, L. Wang, and M. Jiang, MIMO-OFDM for LTE, WIFI and WIMAX: Coherent versus Non-Coherent and Cooperative Turbo-Transceivers. New York, NY, USA: Wiley, 2010.

2] F. Verde, T. Korakis, E. Erkip, and A. Scaglione, "A simple recruitment scheme of multiple nodes for cooperative MAC," IEEE Trans. Commun., vol. 58, no. 9, pp. 2667-2682, Sep. 2010.

[3] G. N. Shirazi, P. Y. Kong, and C. K. Tham, "Distributed reinforcement learning frameworks for cooperative retransmission in wireless networks," IEEE Trans. Veh. Technol., vol. 59, no. 8, pp. 4157-4162, Oct. 2010.

4] T. Luo, V. Srinivasan, and M. Motani, "A metric for DISH networks: Analysis, implications, and applications," IEEE Trans. Mobile Comput., vol. 9, no. 3, pp. 376-389, Mar. 2010.

[5] A. Argyriou, "Cross-layer and cooperative opportunistic network coding in wireless ad hoc networks," IEEE Trans. Veh. Technol., vol. 59, no. 2, pp. 803-812, Feb. 2010

6] H. Shan, W. Zhuang, and Z. Wang, "Distributed cooperative MAC for multihop wireless networks," IEEE Commun. Mag., vol. 47, no. 2, pp. 126-133, Feb. 2009.

7] P. Liu, Z. Tao, S. Narayanan, T. Korakis, and S. S. Panwar, "CoopMAC: A cooperative MAC for wireless LANs," IEEE J. Sel. Areas Commun., vol. 25, no. 2, pp. 340-354, Feb. 2007.

[8] J. Feng, R. Zhong, S. X. Ng, and L. Hanzo, "Relay selection for energy-efficient cooperative media access control," in Proc. IEEE WCNC, Cancun, Mexico, Mar. 2011, pp. 287-292.

9] Z. Zhou, S. Zhou, J. Cui, and S. Cui, "Energy-efficient cooperative communication based on power control and selective single-relay in wireless sensor networks," IEEE Trans. Commun., vol. 7, no. 8, pp. 3066-3078, Aug. 2008.

10] S. Mehta and K. S. Kwak, "An energy-efficient MAC protocol in wireless sensor networks: A game theoretic approach," EURASIP J. Wireless Commun. Netw., vol. 2010, pp. 1-10, May 2010.

1] K. B. Letaief and W. Zhang, "Cooperative communications for cognitive radio networks," Proc. IEEE, vol. 97, no. 5, pp. 878-893, May 2009.

2] R. Madan, N. B. Mehta, A. F. Molisch, and J. Zhang, "Energy-efficient cooperative relaying over fading channels with simple relay selection," IEEE Trans. Commun., vol. 7, no. 8, pp. 3013-3025, Aug. 2008.

3] T. Luo, M. Motani, and V. Srinivasan, "Energy-efficient strategies for cooperative multi-channel MAC protocols," IEEE Trans. Mobile Comput., vol. 11, no. 4, pp. 553-566, Apr. 2012.

14] Y. Zhou, J. Liu, C. Zhai, and L. Zheng, "Two-transmitter two-receiver cooperative MAC protocol: Cross-layer design and performance analysis," IEEE Trans. Veh. Technol., vol. 59, no. 8, pp. 4116-4127, Oct. 2010.

5] Q. Zhao and B. Sadler, "A survey of dynamic spectrum access," IEEE Signal Proc. Mag., vol. 24, pp. 79-89, May 2007.

6] A. Mukherjee and H. M. Kwon, "General auction-theoretic strategies for distributed partner selection in cooperative wireless networks," IEEE Trans. Commun., vol. 58, no. 10, pp. 2903-2915, Oct. 2010.
[17] I. Stanojev, O. Simeone, U. Spagnolini, Y. Bar-Ness, and R. Pickholtz, 751 "Cooperative ARQ via auction-based spectrum leasing," IEEE Trans. 752 Commun., vol. 58, no. 6, pp. 1843-1856, Jun. 2010.

[18] F. Pantisano, M. Bennis, W. Saad, and M. Debbah, "Spectrum leasing as 754 an incentive towards uplink macrocell and femtocell cooperation," IEEE 755 J. Sel. Areas Commun., vol. 30, no. 3, pp. 617-630, Apr. 2012.

[19] Asaduzzaman, H. Y. Kong, and I. Koo, "Opportunistic relaying based 757 spectrum leasing for cognitive radio networks," J. Commun. Netw., 758 vol. 13, no. 1, pp. 50-55, Feb. 2011.

[20] M. Hafeez and J. Elmirghani, "Analysis of dynamic spectrum leasing 760 for coded bi-directional communication," IEEE J. Sel. Areas Commun., 761 vol. 30, no. 8, pp. 1500-1512, Sep. 2012.

762

[21] S. Jayaweera, M. Bkassiny, and A. Very, "Asymmetric cooperative com- 763 munications based spectrum leasing via auctions in cognitive radio net- 764 works," IEEE Trans. Wireless Commun., vol. 10, no. 8, pp. 2716-2724, 765 Aug. 2011.

[22] IEEE Standard for Information Technology-Telecommunications and 767 Information Exchange Between Systems-Local and Metropolitan Area 768 Networks-Specific Requirements-Part 11: Wireless LAN Medium Access 769 Control (MAC) and Physical Layer (PHY) Specifications, IEEE Std. 770 802.11 , 2007. [Online]. Available: http://standards.ieee.org/getieee802/ 771 802.11.html

[23] D. Chase, "Digital signal design concepts for a time-varying Rician 773 channel," IEEE Trans. Commun., vol. COMM-24, no. 2, pp. 164-172, 774 Feb. 1976.

[24] I. Stanojev, O. Simeone, Y. Bar-ness, and C. You, "Performance of multi- 776 relay collaborative hybrid-ARQ protocols over fading channels," IEEE 777 Commun. Lett., vol. 10, no. 7, pp. 522-524, Jul. 2006.

[25] L. Hanzo, M. Munster, B. Choi, and T. Keller, OFDM and MC-CDMA 779 for Broadcasting Multi-User Communications, WLANs and Broadcasting. 780 Chichester, U.K.: Wiley, 2003.

[26] J. G. S. Cui and A. Bahai, "Energy-constrained modulation optimization," 782 IEEE Trans. Commun., vol. 4, no. 5, pp. 2349-2360, Sep. 2005.

[27] X. Gao, B. Jiang, Z. P. X. You, Y. Xue, and E. Schulz, "Efficient channel 784 estimation for MIMO single-carrier block transmission with dual cyclic 785 timeslot structure," IEEE Trans. Commun., vol. 55, no. 11, pp. 2210- 786 2223, Nov. 2007.

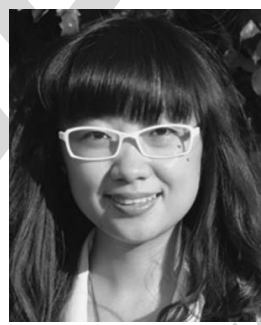

Jiao Feng received the B.Eng. degree and the M.Sc. 788 degree in communications engineering from Jilin 789 University, Jilin, China, in 2007 and 2009, respec- 790 tively. She is currently working toward the Ph.D. 791 degree in wireless communications with the School 792 of Electronics and Computer Science, University of 793 Southampton, Southampton, U.K., under the sup- 794 port of the China-U.K. Excellence Scholarship from 795 both the University of Southampton and the China 796 Scholarship Council.

797

Her research interests include cooperative com- 798 munication, medium-access-control-layer protocols, and cognitive radio 799 networks.

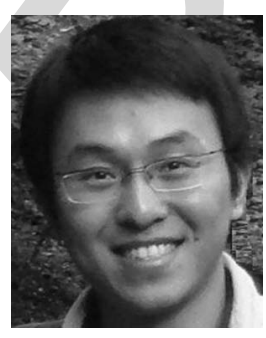

Rong Zhang (M'09) received the B.Sc. degree 801 from Southeast University, Nanjing, China, in 2003802 and the Ph.D. degree from the University of 803 Southampton, Southampton, U.K., in 2009.

From August 2003 to July 2004, he was an En- 805 gineer with China Telecom, Beijing, China. From 806 January 2006 to May 2009, he was a Research As- 807 sistant with the Mobile Virtual Center of Excellence, 808 Hampshire, U.K. From August 2009 to July 2012, he 809 was a Postdoctoral Researcher with the University of 810 Southampton. From August 2012 to January 2013, 811 he took his industrial consulting leave for Huawei Sweden Research and 812 Development as a System Algorithms Specialist. Since February 2013, he has 813 been a Lecturer with the Communications, Signal Processing, and Control 814 Group, Electronics and Computer Science, University of Southampton. He is 815 also a Visiting Researcher with Nanjing University, Nanjing, China, under the 816 Worldwide University Network. He is the author several papers in more than 817 25 journals in prestigious publication avenues [e.g., the IEEE and The Optical 818 Society] and in major conference proceedings.

Dr. Zhang regularly serves as a Reviewer for IEEE TRANSACTIONS/ 820 JouRnAls, and he has been a Technical Program Committeee Member/Invited 821 Session Chair of major conferences several times. He received joint funding 822 from the MVCE and the Engineering and Physical Sciences Research Council. 823 


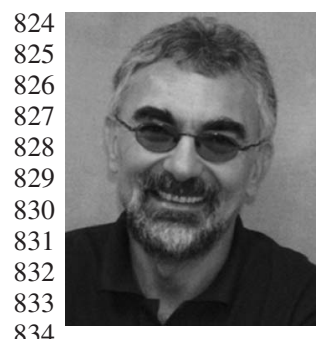

Lajos Hanzo (M'91-SM'92-F'04) received the M.S. degree (with first-class honors) in electronics and the Ph.D. degree from the Technical University of Budapest, Budapest, Hungary, in 1976 and 1983, respectively, the D.Sc. degree from the University of Southampton, Southampton, U.K., in 2004, and the "Doctor Honoris Causa" degree from the Technical University of Budapest in 2009.

During his 35-year career in telecommunications, he has held various research and academic posts in Hungary, Germany, and the U.K. Since 1986, he has 835 been with the School of Electronics and Computer Science, University of 836 Southampton, Southampton, U.K., where he holds the Chair for Telecommuni837 cations. Since 2009, he has been a Chaired Professor with Tsinghua University, 838 Beijing China. He is currently directing a 100-strong academic research team, 839 working on a range of research projects in the field of wireless multimedia 840 communications sponsored by industry; the Engineering and Physical Sciences 841 Research Council, U.K.; the European IST Programme; and the Mobile Virtual 842 Centre of Excellence, U.K. He is an enthusiastic supporter of industrial and 843 academic liaison and offers a range of industrial courses. He has successfully 844 supervised 80 Ph.D. students, coauthored 20 John Wiley/IEEE Press books on 845 mobile radio communications totaling in excess of 10000 pages, published 846 more than 1250 research entries on IEEE Xplore, and presented keynote 847 lectures. (For further information on research in progress and associated 848 publications, please refer to http://www-mobile.ecs.soton.ac.uk.)

849 Dr. Hanzo is Fellow of the Royal Academy of Engineering, U.K., a Fellow 850 of the Institution of Electrical Engineers, and a Governor of the IEEE Vehicular 851 Technology Society. He has been a Technical Program Committee Chair and a 852 General Chair for IEEE conferences. During 2008-2012, he was the Editor-in853 Chief of the IEEE Press. He has received a number of distinctions.

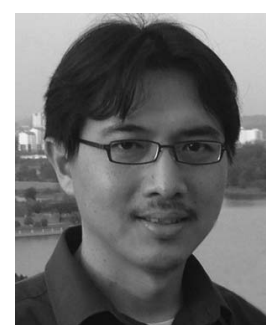

Soon Xin Ng (S'99-M'03-SM'08) received the 854 B.Eng. degree (first-class honors) in electronics en- 855 gineering and the Ph.D. degree in wireless com- 856 munications from the University of Southampton, 857 Southampton, U.K., in 1999 and 2002, respectively. 858 From 2003 to 2006, he was a Postdoctoral Re- 859 search Fellow working on collaborative European 860 research projects known as SCOUT, NEWCOM, 861 and PHOENIX. Since August 2006, he has been a 862 member of the academic staff with Electronics and 863 Computer Science, University of Southampton. He 864 is involved in the OPTIMIX and CONCERTO European projects and the IU- 865 ATC and UC4G projects. He is currently a Senior Lecturer with the University 866 of Southampton. He is the author of over 150 papers and a coauthor of two 867 John Wiley/IEEE Press books in his field of expertise. His research inter- 868 ests include adaptive coded modulation, coded modulation, channel coding, 869 space-time coding, joint source and channel coding, iterative detection, orthog- 870 onal frequency-division multiplexing, multiple-input-multiple-output systems, 871 cooperative communications, distributed coding, quantum error correction 872 codes, and joint wireless-and-optical-fiber communications.

Dr. $\mathrm{Ng}$ is a Chartered Engineer and a Fellow of the Higher Education 874 Academy in the U.K. 


\section{AUTHOR QUERIES}

\section{AUTHOR PLEASE ANSWER ALL QUERIES}

AQ1 = Please provide keywords.

AQ2 = Please provide expanded form of OF.

AQ3 = What is the first initial of author Asaduzzaman?

\section{END OF ALL QUERIES}

